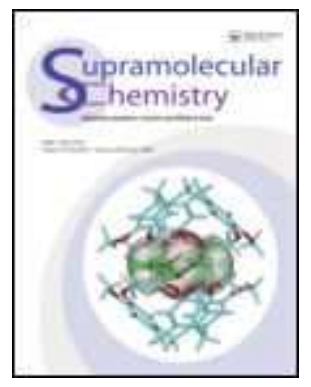

\title{
Novel Adamantane-Bearing Anilines and Properties of Their Supramolecular Complexes with $\beta$-Cyclodextrin
}

\begin{tabular}{|c|c|}
\hline Journal: & Supramolecular Chemistry \\
\hline Manuscript ID: & GSCH-2010-0205.R1 \\
\hline Manuscript Type: & Full Paper \\
\hline $\begin{array}{r}\text { Date Submitted by the } \\
\text { Author: }\end{array}$ & 06-May-2011 \\
\hline Complete List of Authors: & $\begin{array}{l}\text { Vicha, Robert; Tomas Bata University in Zlin, Faculty of Technology, } \\
\text { Department of Chemistry } \\
\text { Rouchal, Michal; Tomas Bata University in Zlin, Faculty of } \\
\text { Technology, Department of Chemistry } \\
\text { Kozubková, Zuzana; Tomas Bata University in Zlin, Faculty of } \\
\text { Technology, Department of Chemistry } \\
\text { Kuřitka, Ivo; Tomas Bata University in Zlin, Faculty of Technology, } \\
\text { Polymer Centre } \\
\text { Marek, Radek; Masaryk University, National Centre for Biomolecular } \\
\text { Research } \\
\text { Branná, Petra; Tomas Bata University in Zlin, Faculty of } \\
\text { Technology, Department of Chemistry } \\
\text { Čmelík, Richard; Institute of Analytical Chemistry of the ASCR, } \\
\text { v.v.i. }\end{array}$ \\
\hline Keywords: & adamantane, amines, cyclodextrins, host-guest systems \\
\hline \multicolumn{2}{|c|}{$\begin{array}{l}\text { Note: The following files were submitted by the author for peer review, but cannot be converted } \\
\text { to PDF. You must view these files (e.g. movies) online. }\end{array}$} \\
\hline $\begin{array}{l}\text { Scheme_1.cdx } \\
\text { Scheme_2.cdx } \\
\text { Scheme_3.cdx }\end{array}$ & \\
\hline
\end{tabular}


2

3

4

5

6

7

8

9

10

11

12

13

14

15

16

17

18

19

20

21

22

23

24

25

26

27

28

29

30

31

32

33

34

35

36

37

38

39

40

41

42

43

44

45

46

47

48

49

50

51

52

53

54

55

56

57

58

59

60

\section{SCHOLARONE ${ }^{\text {m }}$ \\ Manuscripts}




\section{Novel Adamantane-Bearing Anilines and Properties of Their Supramolecular}

\section{Complexes with $\boldsymbol{\beta}$-Cyclodextrin}

Robert Vícha ${ }^{a, *}$, Michal Rouchal ${ }^{a}$, Zuzana Kozubkováa ${ }^{a}$ Ivo Kuritka $^{b}$, Radek Marek $^{c, d}$, Petra Brannáa and Richard Čmelík ${ }^{e}$

a) Department of Chemistry, Faculty of Technology, Tomas Bata University in Zlin, Náměstí T. G. Masaryka 275, 76001 Zlin, Czech Republic ${ }^{b}$ ) Polymer Centre, Faculty of Technology, Tomas Bata University in Zlin, Náměstí T. G. Masaryka 275, 76001 Zlín, Czech Republic c) National Centre for Biomolecular Research, Masaryk University, Kamenice 5/A4, 62500 Brno, Czech Republic ${ }^{d}$ ) Central European Institute of Technology (CEITEC), Masaryk University, Kamenice 5/A4, 62500 Brno, Czech Republic ${ }^{e}$ ) Institute of Analytical Chemistry of the ASCR, v.v.i., Veveři 97, 60200 Brno, Czech Republic
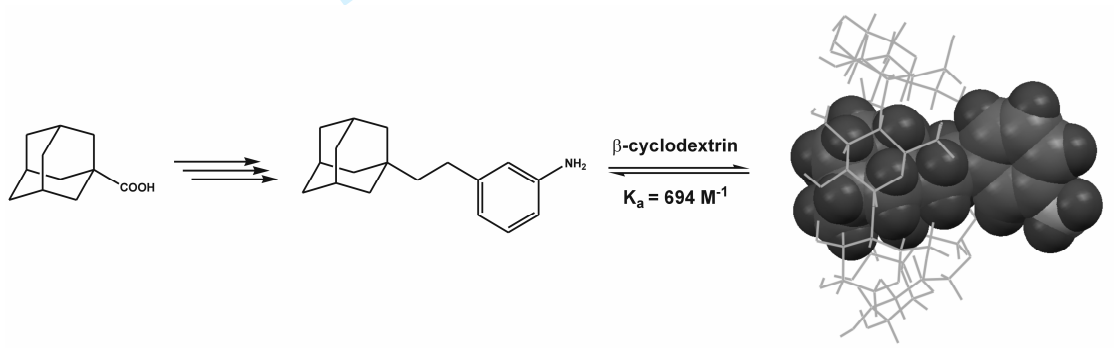
1

2

3

4

5

6

7

8

9

10

11

12

13

14

\section{Novel Adamantane-Bearing Anilines and Properties of Their Supramolecular Complexes with $\beta$-Cyclodextrin}

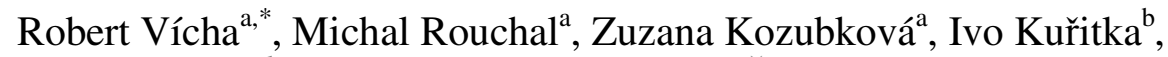
Radek Marek ${ }^{\mathrm{c}, \mathrm{d}}$, Petra Branná and Richard Čmelík ${ }^{\mathrm{e}}$

Deleted: $\check{C}$ elík ${ }^{d}$

a) Department of Chemistry, Faculty of Technology, Tomas Bata University in Zlin,

Náměstí T. G. Masaryka 275, 76001 Zlín, Czech Republic

b) Polymer Centre, Faculty of Technology, Tomas Bata University in Zlin, Náměstí T.

G. Masaryka 275, 76001 Zlín, Czech Republic

$\left.{ }^{c}\right)$ National Centre for Biomolecular Research, Masaryk University, Kamenice 5/A4, 62500 Brno, Czech Republic

${ }^{\mathrm{d})}$ Central European Institute of Technology (CEITEC), Masaryk University,

Kamenice 5/A4, 62500 Brno, Czech Republic

e) Institute of Analytical Chemistry of the ASCR, v.v.i., Veveři 97, 60200 Brno, Czech Republic

*Corresponding author: Tel: +420-576031003; fax: +420-576031560; e-mail: rvicha@ft.utb.cz
Formatted: Superscript

Formatted: Normal

Deleted: $^{\mathrm{d}}$ 


\title{
Novel Adamantane-Bearing Anilines and Properties of Their Supramolecular Complexes with $\beta$-Cyclodextrin
}

\begin{abstract}
Several novel anilines bearing 1-adamantyl substituents that are useful for drug modification were synthesised from the corresponding 1-adamantyl (nitrophenyl) ketones. The host-guest systems of these prepared ligands with $\beta$ cyclodextrin were studied using ESI-MS, NMR spectroscopy, titration calorimetry, and semi-empirical calculations. The complexes with 1:1 stoichiometry were found to predominantly exist as pseudorotaxane-like threaded structures with the adamantane cage sitting deep in the cavity of $\beta$ cyclodextrin close to the wider rim. Such geometry was observed for all examined amines and is independent of their structure and/or presence of protic substituents.
\end{abstract}

Keywords: adamantane; amines; cyclodextrins; host-guest systems

\section{Introduction}

Since the first description of the antiviral activity of 1-adamantylamine in $1964,{ }^{1}$

various compounds containing the adamantane scaffold have been shown to exhibit antiviral, $^{2}$ anticancer ${ }^{3}$ and antimicrobial ${ }^{4}$ activities; such compounds have also been described as hypoglycaemic, ${ }^{5}$ proapoptotic, ${ }^{6}$ and neuroprotective ${ }^{7}$ agents, as well as possible treatments for hypertension, vascular inflammation ${ }^{8}$ and tuberculosis ${ }_{-v}^{9}$ Adamantane-bearing compounds can also serve as cannabinoid receptor ligands. ${ }^{10}$ This well-founded interest is related to a unique property of the adamantane cage that can improve the characteristics of biologically active compounds. As a result of its high lipophilicity, adamantane should increase the rate of transfer of a modified drug through cell membranes and thus facilitate the distribution of the drug. On the other hand, the formation of supramolecular complexes with $\beta$-cyclodextrin $(\beta-C D)^{11}$ significantly increases drug's solubility in water. Cyclodextrin drug carrier systems have been studied extensively in terms of solubility, bioavailability and stability. ${ }^{12}$ This attention has yielded several commercial pharmaceutical products based on cyclodextrin host-guest complexes. ${ }^{12 \mathrm{~b}}$ The adamantane-bearing amines are a suitable 


\section{candidates for drug modification e.g. as a ligands in preclinically tested ${ }^{3 \mathrm{~b}}$ platinum derivate LA-12 (Figure 1, left) or as a building blocks for displacement of C6 $\underline{\text { substituent in purvalanol-like promising anticancer drugs (Figure 1, right). However, }}$} the steric hindrance of bulky adamantane may lead to attenuation of the desired activity if the scaffold is introduced too close to the active site of the drug ${ }^{13}$ hence the need for preparation and property investigation of new suitable adamantane-bearing

\section{building blocks is justified.}

While inclusion complexes of $\beta$-CD with 1-adamantyl-based compounds have been studied for a long time, previous efforts have focused on small ionic guests. ${ }^{14}$ Some structural data have also been published for more complex ligands. ${ }^{15}$ In most of these cases, the nature of the inclusion complexes is determined by the structure of host and/or guest molecule. It is reasonable to suppose that the geometry and stability of host-guest complexes are affected by substituents adjacent to the adamantane cage. Therefore, we have prepared several new potential building blocks with modulated polarity and variable linker length between the adamantane and benzene ring units. The host-guest complexes of these prepared anilines and $\beta-\mathrm{CD}$ were investigated using electrospray ionisation mass spectrometry (ESI-MS), ${ }^{1} \mathrm{H}$ and ${ }^{13} \mathrm{C}$ NMR spectroscopy, and titration calorimetry.

\section{Results and Discussion}

\subsection{Synthesis of amines}

The nitro intermediates were prepared following previously described procedures, including the ketone preparation ${ }^{16}$ and nitration with acetyl nitrate ${ }^{17}$ shown in Scheme 1. Regioisomers were separated by column chromatography, and compounds $3-5$ were used as starting materials for further reactions.
Deleted: , but

Deleted: 
Aminoketones 6-8 were prepared in methanolic $\mathrm{HCl}$ solution using iron powder as a reducing agent. The iron powder used in this reaction was obtained from iron pentacarbonyl decomposition (purchased from commercial source); use of iron fillings or turnings led to considerably longer reaction times. Amines 6-8 are rather unstable at room temperature as a free base (but may be stored for several months at $10^{\circ} \mathrm{C}$ ) and decompose to dark brown oily products within a few days. Unfortunately, transformation to their corresponding solid hydrochloride salts via introduction of dry gaseous hydrogen chloride into diethyl ether or hexane solution only provided oily, brownish products.

Aminoalcohols 13-15 were prepared from nitroketones in two steps. Selective reduction using $\mathrm{NaBH}_{4}$ proved to be very effective in our case, and we obtained nitroalcohols 9-11 in excellent yields ( 95\%) in $30 \mathrm{~min}$. Reduction of the nitro group was carried out using iron powder in a methanol/ $\mathrm{HCl}(1 / 1, \mathrm{v} / \mathrm{v}$; conc. $\mathrm{HCl}$ was used) mixture. Amines $\mathbf{1 3}$ and $\mathbf{1 4}$ were isolated either as free bases ( $\mathrm{pH}$ adjustment followed by extraction) or directly as hydrochloride salts. Attempted preparation of aminoalcohol $\mathbf{1 5}$ in the same manner failed due to undesirable nucleophilic substitution, and methoxyamine $\mathbf{1 2}$ was isolated in $85 \%$ yield. Therefore, catalytic hydrogenation on Ra-Ni was employed in the preparation of amine $\mathbf{1 5}$ (Scheme 2). Attempts to prepare aminoalcohols from compounds 3-5 in one step using less selective reducing agents such as $\mathrm{LiAlH}_{4}$ or $\mathrm{H}_{2} / \mathrm{Ra}-\mathrm{Ni}$ were not successful, and complex mixtures were obtained.

Amines with non-polar hydrocarbon spacers between the adamantane and benzene ring moieties (25-27) were also prepared in two (via 1,3-dithianes) or three (via 1,3-dithiolanes) steps. This synthesis involved the formation of the corresponding $S, S$-acetals, followed by reduction of the nitro group by iron powder in alcohol/HCl 


\subsection{ESI-MS analysis}

Solutions of individual amines, as well their 1:1 mixtures with $\beta-\mathrm{CD}$, were studied by

ESI-MS. The dominant ions corresponding to the amines were the pseudomolecular ions $[\mathrm{M}+\mathrm{H}]^{+}$, accompanying the signals at $\mathrm{m} / \mathrm{z}$ values about two times as high $\left(\right.$ exactly $[2 \times(M+H)-1]^{+}$or $\left.[2 \times M+23]^{+}\right)$. The latter ions were observed when a polar functional group (oxo or hydroxy) was present in the amine molecule, as shown for

amine 13 in Figure $2 \mathrm{a}$. These signals are assumed to be related to associates of dimer

Deleted: 1a 
linked via hydrogen bonds with a proton or sodium cation, respectively. The formation of analogous dimers in the solid state has been observed for aminoalcohol 15. ${ }^{18}$ In the amine/ $\beta-C D$ mixtures, the protonated amine and sodium adduct of $\beta-C D$, as well as protonated $\beta-C D$-amine complex, were detected for all examined amines (Table 1). Figure $2 \mathrm{~b}$ shows a typical spectrum of an equimolar mixture of $\beta-\mathrm{CD}$ and Deleted: $1 b$ amine 27. The tandem mass spectrum of the protonated complex showed a characteristic fragmentation pattern, which confirms its identity. The ions at $\mathrm{m} / \mathrm{z}$ $1136,974,811,649$, and 487 resulted from the successive losses of amine and glucose residues of the $\beta$-CD moiety (Figure $2 \mathrm{c}$ ).

\subsection{The geometry of host-guest complexes}

The $\beta$-CD is a heptamer built up from glucopyranose units linked by $\alpha-1,4$-glycosidic bonds with a very well-known structure ${ }^{19}$ that it is often described as a doughnut with rims of differing diameters. The larger diameter corresponds to the secondary rim where secondary hydroxyl groups at $\mathrm{C} 2$ and $\mathrm{C} 3$ are located; primary hydroxyl groups at $\mathrm{C} 6$ are placed on the opposite smaller primary rim due to the non-alternating orientation of the glucose units. The interior of the cavity has steric constraints due to $\mathrm{H} 3$ and $\mathrm{H} 5$ protruding into the cavity. ${ }^{14 \mathrm{c}}$ Schematics with the relevant dimensions of $\beta-\mathrm{CD}$ and the prepared amines are displayed in Figure 3.

The internal diameter of the cavity is likely to be slightly smaller than the diameter of the nearly ball-shaped adamantane moiety, which cannot pass through it easily but still fit well the interior of the $\beta$-CD cavity, As a result, two distinct complexes may form. The adamantane moiety can be located either at the primary rim would fit well inside the region or at the secondary rim region. In solution, a reasonable orientation of a short and, in most cases, charged, substituent bound to adamantane is outside the $\beta$-CD cavity. ${ }^{14 c, 15 a}$ Occupancy of the primary rim was observed only when the secondary 
1

2

3

4

5

6

7

8

9

10

rim was blocked. Higher thermodynamic stabilities were calculated for complexes with the adamantane unit sitting in the secondary rim. ${ }^{14 c}$ However, it is reasonable to suppose that a non-polar substituent of appropriate length may thread through the cavity of $\beta$-CD. Thus, four possible arrangements of 1:1 adamantane and $\beta$-CD complexes should be considered. These arrangements are illustrated in Figure 4.

All examined systems obey the fast exchange mode on the NMR time scale, and thus only one set of signals was observed in all cases. Unfortunately, the shifts observed upon complexation for the host and guest protons were small $\left(\leq 10^{-2} \mathrm{ppm}\right)$, and determination of thermodynamic parameters from NMR titrations was generally Deleted: of $10^{-3}-$ impossible. We observed reproducible complexation-induced shifts of well-resolved ${ }^{1} \mathrm{H}$ NMR signals only for guest $\mathbf{1 4}$. Although the Job plot for the H5 protons (Figure Deleted: S1) of the adamantane guest indicates a 1:1 stoichiometry, the analysis of titration data was unsatisfactory. The fitting of experimental data to the theoretical rectangular hyperbola using the standard least square regression procedure (MicroCal ORIGIN) led to estimation of association constant being $\sim 40 \mathrm{M}^{-1}$, but the systematic discrepancy between the theoretical data and best fit curve is too high (Figure S2). We attribute this discrepancy to the influence of higher ordered, hydrogen-bonded complexes on the observed chemical shifts. Nevertheless, the down-field shifts for guest protons H4-6 (on the adamantane cage) and up-field shifts for $\mathrm{H} 1, \mathrm{H}_{2}^{\mathrm{A}, \mathrm{B}}$, H14, and H16-18 were clearly observed (Figure S3).

The observed nOe interactions between guest protons bound to the adamantane cage (H4-6 for guest $\mathbf{1 4}$ ) and inner hydrogen atoms of the cyclodextrin cavity suggest the formation of an inclusion complex with adamantane positioned inside the $\beta$-CD cavity. The observation of relatively strong nOe interactions between guest protons $\mathrm{H} 2$ and $\beta-\mathrm{CD}-\mathrm{H} 5$, together with weak (if any) interactions with $\beta$-CD- 
$\mathrm{H} 3$, indicates the occupancy of the secondary rim of $\beta-\mathrm{CD}$ by the adamantane cage. Additionally, in the case of all hydroxylated guests (13-15), the nOe interactions between the inner $\beta$-CD hydrogen atoms and $\mathrm{H} 6_{\mathrm{ax}}$ of adamantane are significantly weakened or completely missing from the spectra, whereas those with $\mathrm{H}_{\mathrm{eq}}$ are observed. A portion of the NOESY spectrum of a mixture of amine $\mathbf{1 4}$ and $\beta$ cyclodextrin is shown in Figure 5 (left). However, ${ }^{1} \mathrm{H}$ NMR signals of $\beta-\mathrm{CD}$ inner protons $\mathrm{H} 3$ and protons $\mathrm{H} 6$ of the secondary rim were significantly overlapped in DMSO solution, and interpretation of the observed cross-peaks in standard NOESY spectrum became ambiguous. Therefore, we applied a $2 \mathrm{D}{ }^{1} \mathrm{H}-{ }^{13} \mathrm{C}$ gs-HMQC-NOESY experiment to increase the spectral resolution by employing a carbon frequency in an indirect dimension to assign the individual nOe contacts unequivocally. A schematic of the host-guest complex 7- $\beta$-CD and its observed interactions are depicted in Figure

5 (middle and right). Both the observed interactions of adamantane protons $\mathrm{H} 4$ and H5 with $\beta$-CD carbons $\mathrm{C} 3$ and the absence of interactions between these same protons and $\beta-\mathrm{CD}$ carbons $\mathrm{C} 5$ indicate a positioning of the adamantane cage inside the $\beta-\mathrm{CD}$ cavity with bridgehead-substituted carbon $\mathrm{C} 2$ located close to the secondary rim of $\beta$ CD. In addition, observed interactions of phenyl protons $\mathrm{H} 13$ and $\mathrm{H} 17$ with $\beta-\mathrm{CD}$ carbons C6 and C5 support the proposed structural model in which the aromatic part of the guest protrudes from the secondary rim of $\beta$-CD (Figure 5). According to the notation in Figure 4 , the observed arrangement of the examined host-guest complexes is assigned as SI.

The binding properties of prepared guests $6-8, \mathbf{1 3 - 1 5}$, and 25-27 were studied using isothermal titration calorimetry. All three aminoalcohols 13-15 exhibited additional heat release during both titration and dilution experiments; therefore, thermodynamic parameters were not able to be determined. This observation may be 
1

2

3

4

5

6

7

8

9

10

11

12

13

14

reasonably attributed to additional equilibria related to the dissociation of dimers and/or higher associates of guest molecules. In addition, dilution data of these aminoalcohols did not fit the theoretical curve using a simple "dissociation" model, which takes into account only dimer dissociation. Therefore, it is reasonable to assume additional equilibria involving higher-ordered associations. Anilines $\mathbf{2 5}$ and 27 exhibited some exothermic process that very slowly equilibrated. This slow equilibration thwarted the collection of usable data. The obtained values of binding constants, enthalpies, entropies and stoichiometries of the complexes for aminoketones 6-8 and amine 26 are listed in Table 2. For the typical raw data, integrated values of heat released and the fitted curve for guest $\mathbf{7}$, see Supplementary data, Figure $\$ 4$.

\subsection{Computation}

In order to support the structural conclusions about host-guest complexes formulated from NMR analysis, we performed the modeling of these complexes for amines $\mathbf{1 4}$ and 26 with $\beta-C D$ at a semi-empirical level of theory. Semi-empirical PM3 method $^{20}$ proved to perform well across a diverse group of macrocycles, particularly for CDs. ${ }^{21}$ Moreover, the PM3 method was selected among available semi-empiricals because of its superiority to AM1 in dealing with hydrogen bonded molecules $\frac{22}{2}$ Although PM3 method chosen for our preliminary calculations appeared to be a powerful tool in conformational studies of supramolecular systems, computed relative energies should be handled with the full awareness of the weakness of semi-empiricals in relative energy estimations and interpreted along with corroborating experimental data. An exhaustive, up-to-date theoretical study may require equilibrium geometries generated by PM3, combined with single point energy calculations at higher levels of theory, preferably DFT which accounts thermochemistry better than semi-empirical methods, 
as these sequential methods are reported in recent literature and successfully applied for CDs $\frac{23}{v_{-}}$

A series of calculations, described in detail in the Experimental section, yielded geometries and energies for several examined positions. The most energetically favoured geometries for amines $\mathbf{1 4}$ and $\mathbf{2 6}$, for both directions of their virtual threading through the $\beta$-CD cavity, are depicted in Figure 6 , and selected geometric parameters and energies are collected in Table 2. In the case of amine 14, the adamantane cage occupies the secondary or primary rim with distances of $C g 1-$ $O g$ being shorter than $0.16 \mathrm{~nm}$, with the benzene ring positioned on the opposite side of the $\beta-C D$. In respect to the orientations defined in Figure 4 , they may be called as SI and PI, respectively. In the case of amine $\mathbf{2 6}$, the respective threading resulted in geometries with adamantane located close to the secondary rim with the $C g 1-O g$ being shorter than $0.12 \mathrm{~nm}$, i.e. SE and SI. For both examined amines, the complex with SI geometry was the most populated in the thermodynamic equilibrium.

The calculations were performed for molecules in vacuo, neglecting the fact that complex formation might be driven by differences in solvation energies of the host-guest complex and its building blocks. Moreover, the large number of possible orientations of the $\beta$-CD's hydroxyl groups is beyond our consideration. Although only one initial conformation of the cyclodextrin was used for each minimisation, the method provides a consistent indicator of the hydrogen bond stabilisation effect as well. To assess the importance of hydrogen bonding in the complex formation, a modelling experiment was performed. Non-polar parent hydrocarbon $(\mathrm{PH})^{24}$ was virtually threaded through the $\beta$-CD's cavity in the same way as described above with the amines. Hence, we obtained analogous results for $\mathbf{P H}$ and amines $\mathbf{1 4}$ and 26, as shown in Table 3. Although partial stabilisation of this complex geometry via 
1

2

3

4

5

6

7

8

9

10

11

12

13

14

15

16

17

18

19

20

21

22

23

24

25

26

27

28

29

30

31

32

33

34

35

intermolecular hydrogen bonds was expected, it is not clearly manifested here.

Therefore, we suggest only a small contribution of intermolecular H-bonds to the stabilisation of the complex in the gas phase.

\section{Conclusions}

Ten new anilines bearing adamantane with linkers of varying polarity and length were synthesised and fully characterised using spectral methods. The inclusion complexes of these anilines with $\beta$-CD were detected using ESI-ion trap MS, and their structures were determined by 2D NMR experiments in DMSO. In agreement with our experimental NMR data and molecular modelling, the most populated inclusion complex between $\beta$-CD and adamantane guests with a long, uncharged substituent may be characterised as a pseudorotaxane-like structure in which the adamantane group is sitting deep in the $\mathrm{CD}$ cavity close to the wider secondary rim of the $\beta$-CD and the substituent protrudes from the primary rim. Association constants of the prepared amines and $\beta-\mathrm{CD}$ were estimated to be on the order of $10^{2} \mathrm{M}^{-1}$ by isothermal calorimetric titrations. These binding properties allowed us to consider the use of these prepared amines in further research on drug modification.

\section{Experimental Section}

\subsection{General}

All starting compounds, reagents and solvents were purchased from commercial sources in analytical quality and were used without further purification. Adamantane1-carbonyl chloride ${ }^{16}$ was prepared following a previously published procedure. Melting points were measured on a Kofler block and are uncorrected. Elemental analyses (C, H, N, S) were performed on a Thermo Fisher Scientific Flash EA 1112. Retention times were determined using TLC plates (Alugram Sil G/UV) from Machrey-Nagel and petroleum ether/ethyl acetate as mobile phase. Three 
compositions of mobile phases were used (v/v): system a (1/1), system b (4/1) and system c (8/1). NMR spectra were recorded on a Bruker Avance 500 spectrometer operating at frequencies of $500.13 \mathrm{MHz}\left({ }^{1} \mathrm{H}\right)$ and $125.77 \mathrm{MHz}\left({ }^{13} \mathrm{C}\right)$ and a Bruker Avance 300 spectrometer operating at frequencies of $300.13 \mathrm{MHz}\left({ }^{1} \mathrm{H}\right)$ and 75.77 $\mathrm{MHz}\left({ }^{13} \mathrm{C}\right) .{ }^{1} \mathrm{H}$ - and ${ }^{13} \mathrm{C}-\mathrm{NMR}$ chemical shifts were referenced to the signal of solvent $\left({ }^{1} \mathrm{H}: \delta\left(\right.\right.$ residual $\left.\mathrm{CHCl}_{3}\right)=7.27 \mathrm{ppm}, \delta\left(\right.$ residual DMSO- $\left.d_{5}\right)=2.50 \mathrm{ppm} ;{ }^{13} \mathrm{C}:$

$\left.\delta\left(\mathrm{CDCl}_{3}\right)=77.23 \mathrm{ppm}, \delta\left(\mathrm{DMSO}-d_{6}\right)=39.52 \mathrm{ppm}\right)$. The mixing time for $\mathrm{NOESY}^{25}$ experiment was adjusted to $500 \mathrm{~ms}$, and the spin-lock for ROESY was adjusted to 400 ms. The assignment of ${ }^{1} \mathrm{H}$ signals for $\beta-\mathrm{CD}$ was described previously ${ }^{19} \mathrm{c}$ ${ }^{13} \mathrm{C}$ gs-HMQC-NOESY spectrum ${ }^{26}$ was measured at resonance frequencies of 600.15 Deleted: NOESY ${ }^{28}$ $\mathrm{MHz}\left({ }^{1} \mathrm{H}\right)$ and $150.67 \mathrm{MHz}\left({ }^{13} \mathrm{C}\right)$. The HMQC step was adjusted for ${ }^{1} J_{\mathrm{H}-\mathrm{C}}=145 \mathrm{~Hz}$ with a subsequent nOe transfer of $700 \mathrm{~ms}$. The spectrum was recorded in phasesensitive mode using the echo-antiecho protocol ${ }^{27}$. The IR spectra were recorded in a Deleted: $\mathrm{KBr}$ disc with a Mattson 3000 FT-IR instrument. GC-MS analyses were run on a Shimadzu QP-2010 instrument using a Supelco SLB-5ms (30 m, $0.25 \mathrm{~mm}$ ) column. Helium was employed as a carrier gas in a constant linear flow mode $\left(38 \mathrm{~cm} \bullet \mathrm{s}^{-1}\right)$; $100{ }^{\circ} \mathrm{C} / 7 \mathrm{~min}, 25^{\circ} \mathrm{C} / \mathrm{min}$ to $250{ }^{\circ} \mathrm{C}$, hold for the required time. Only peaks of relative abundance exceeding $5 \%$ are listed. The electrospray mass spectra were recorded with an Esquire LC ion-trap mass spectrometer (Bruker Daltonics, Bremen, Germany) equipped with an ESI source. Sample solutions ( $8.8 \mu \mathrm{M}$ in methanol/water $\left.{ }_{2} 1 / 1_{2} \mathrm{v} / \mathrm{v}\right)$ were introduced into the ion source at a flow rate of $3 \mu \mathrm{L} / \mathrm{min}$ via a metal capillary held at high voltage $( \pm 3.5 \mathrm{kV})$. The other instrumental conditions were as follows: drying gas temperature, $250^{\circ} \mathrm{C}$; drying gas flow, $5 \mathrm{dm}^{3} / \mathrm{min}$; nebuliser pressure, 41.37 $\mathrm{kPa}$. Nitrogen was used as both nebulising gas and drying gas. The nozzle-skimmer potential and octopole potential were modified and optimised before each experiment. 
1

2

3

4

5

6

7

8

9

10
Isothermal titration calorimetry measurements were done in a $\mathrm{DMSO} / \mathrm{H}_{2} \mathrm{O}\left(3 / 1_{z_{-}} \mathrm{v}-\mathrm{v}\right)$ solvent mixture using a VP-ITC MicroCal instrument at $30{ }^{\circ} \mathrm{C}$. The concentrations of host in the cell and guest in microsyringe were approximately $7.0 \mathrm{mM}$ and $0.6 \mathrm{mM}$, respectively. The raw experimental data were analysed using MicroCal ORIGIN software. The heats of dilution were taken into account for each guest compound. Data were fitted to a theoretical titration curve using the "one set of binding sites" model.

\subsection{Quantum-Chemical Methods}

All theoretical calculations were carried out using the SPARTAN'08 software package ${ }^{28}$ First, the initial geometries of amines 14 and 26 and $\beta$-CD were optimised with the PM3 method without imposing any symmetrical restrictions. A hypothetical parent hydrocarbon (PH), 1-(1-adamantyl)-2-phenylethane, was used as a non-polar reference to evaluate the effect of hydrogen bonding. This hypothetical parent was constructed and optimised using the same procedure as that used for the amines. The input geometry for the optimisation of $\beta-\mathrm{CD}$ was based on available crystallographic data determined by XRD ${ }^{19 \mathrm{~b}}$ Initial approximations of amine.cyclodextrin complexes were then constructed using the optimised structures of both host and guest molecules. As in the case of their constituents, no restrictions were imposed on the complexes. To characterise the mutual orientations of the molecules, the following values were defined: the centre of mass of the four bridgehead carbons in adamantane skeleton $(C g 1)$, the centre of mass of the seven glycosidic oxygen atoms in $\beta$ cyclodextrin $(\mathrm{Og})$, centre of mass of the six carbons of the benzene ring $(\mathrm{Cg} 2)$, and the best least squares plane of the seven glycosidic oxygen atoms in $\beta$-cyclodextrin $(P)$. The sign in the half-space according to $P$ was defined to be positive close to wider secondary rim and negative close to narrower primary rim. Initially, the amine 
was positioned along the molecular sevenfold axis of $\beta-\mathrm{CD}$ at eleven $C g 1-O g$ distances ranging from 1.0 to $-1.0 \mathrm{~nm}$ in increments of $0.2 \mathrm{~nm}$. The resulting geometries were optimised. These optimised geometries represent the sequential local minima for an amine passing through the CD cavity. The stabilisation energy of complex formation was calculated as the difference between the energy of the complex and the sum of energies of the guest and host calculated independently. The geometry with the absolute minimum energy could in this way be described as the geometry of inclusion complex. On the other hand, the rotation of the guest molecule within the CD's cavity was not tested, as it is known that the optimisation process automatically finds the best relative rotational orientation of the guest and host molecules. ${ }^{29}$ Under real condition, one could expect water filled central cavity in $\underline{\text { CD's that must be displaced if a guest is to enter which would stabilise any interaction }}$ due to hydrophobic effects. Therefore it is expected that basic considerations for this $\underline{\text { virtual experiment are not compromised by neglecting of water molecules in }}$ calculations. Because both the CD and the amine have non-equivalent sides following the threading route, two distinct threading processes must be performed with each amine. It was decided to arrange the threading process as adamantane-on into the secondary and primary rim of the $\mathrm{CD}$, respectively.

\subsection{General procedure for nitro ketones reduction to amino ketones 6-8}

The ketone $(1.05 \mathrm{mmol})$ was dissolved in methanol $(30 \mathrm{~mL})$ and $6 \mathrm{~mL}$ of hydrochloric acid/water (v/v, 1/1) was added. Into the refluxed and well stirred mixture a portions of an iron powder $(2.33 \mathrm{mmol})$ were added successively unless TLC performed after disappearing of each iron portion indicated the consumption of all starting material. The mixture was poured onto a $5 \%$ solution of $\mathrm{NaOH}(40 \mathrm{~mL})$ and extracted several times with diethyl ether. Combined organic layers were washed

Deleted: into 14 
| with brine and dried over sodium sulphate. The crude product was obtained after evaporation of the solvent in vacuo.

4.3.1. 1-Adamantyl-(4-aminophenyl)methanone (6) was purified by column chromatography (silica gel, system a) to yield $257 \mathrm{mg}$ (96\%) of a yellow crystalline powder. Mp $79-81{ }^{\circ} \mathrm{C}, \mathrm{R}_{\mathrm{f}} 0.17$ (system b), anal. calcd for $\mathrm{C}_{17} \mathrm{H}_{21} \mathrm{NO}: \mathrm{C}, 79.96 \% ; \mathrm{H}$, 8.29\%; N 5.49\%; found C, 80.05\%; $\mathrm{H}, 8.12 \%$; $, 5.63 \% .{ }^{1} \mathrm{H} \mathrm{NMR}\left(\mathrm{CDCl}_{3}\right): \delta 1.78$ (m, 6H, $\left.\mathrm{CH}_{2}(\mathrm{Ad})\right), 2.07$ (m, 9H, $\left.\mathrm{CH}_{2}+\mathrm{CH}(\mathrm{Ad})\right), 6.69$ (d, J=8.6 Hz, 2H, Ph), 7.72 (d, $J=8.6 \mathrm{~Hz}, 2 \mathrm{H}, \mathrm{Ph}) \mathrm{ppm} .{ }^{13} \mathrm{C} \mathrm{NMR}\left(\mathrm{CDCl}_{3}\right): \delta 28.6(\mathrm{CH}), 37.0\left(\mathrm{CH}_{2}\right), 39.9\left(\mathrm{CH}_{2}\right)$, $46.9(\mathrm{C}), 114.4(\mathrm{CH}), 129.3(\mathrm{C}), 131.0(\mathrm{CH}), 148.2(\mathrm{C}), 206.5(\mathrm{CO}) \mathrm{ppm}$. IR (KBr): 3469 (m), 3347 (s), 2898 (s), 2847 (m), 1629 (s), 1586 (s), 1557 (m), 1517 (w), 1442 (m), $1322(\mathrm{~m}), 1271(\mathrm{~s}), 1241$ (m), 1171 (s), $1112(\mathrm{~m}), 986(\mathrm{w}), 929(\mathrm{w}), 841(\mathrm{~m}), 751$ (w), 643 (w), 614 (m), 511 (w) cm-1. GC-MS (EI, 70eV); m/z (\%): 65 (8), 79 (9), 92 (9), 93 (7), 120 (100), $121(8), 135(11), 255\left(\mathrm{M}^{+}, 8\right)$.

\subsubsection{1-Adamantyl-(3-aminophenyl)methanone (7) was purified by column}

chromatography (silica gel, system a) to yield $236 \mathrm{mg}$ (88\%) of a colourless crystalline powder. $\mathrm{Mp} 97-100{ }^{\circ} \mathrm{C}, \mathrm{R}_{\mathrm{f}} 0.20$ (system b), anal. calcd for $\mathrm{C}_{17} \mathrm{H}_{21} \mathrm{NO}$ : C, 79.96\%; H, 8.29\%; N, 5.49\%; found C, 79.89\%; H, 8.35\%; N, 5.37. ${ }^{1} \mathrm{H}$ NMR

$\left(\mathrm{CDCl}_{3}\right): \delta 1.75\left(\mathrm{~m}, 6 \mathrm{H}, \mathrm{CH}_{2}(\mathrm{Ad})\right), 1.99\left(\mathrm{~m}, 6 \mathrm{H}, \mathrm{CH}_{2}(\mathrm{Ad})\right), 2.07(\mathrm{~m}, 3 \mathrm{H}, \mathrm{CH}(\mathrm{Ad}))$, 3.73 (bs, 2H, NH $\mathrm{NH}_{2}, 6.72-6.77$ (m, 2H, Ph), 6.91 (d, $\left.J=7.6 \mathrm{~Hz}, 1 \mathrm{H}, \mathrm{Ph}\right), 7.16$ (t, $J=7.6$ $\mathrm{Hz}, 1 \mathrm{H}, \mathrm{Ph}) \mathrm{ppm} .{ }^{13} \mathrm{C} \mathrm{NMR}\left(\mathrm{CDCl}_{3}\right): \delta 28.4(\mathrm{CH}), 36.8\left(\mathrm{CH}_{2}\right), 39.3\left(\mathrm{CH}_{2}\right), 47.1(\mathrm{C})$, $113.7(\mathrm{CH}), 116.8(\mathrm{CH}), 117.3(\mathrm{CH}), 128.9(\mathrm{CH}), 141.2(\mathrm{C}), 146.3(\mathrm{C}), 210.9(\mathrm{CO})$ ppm. IR (KBr): 3474 (m), 3381 (s), 2900 (s), 2850 (m), 1662 (s), 1626 (m), 1593 (m), 1494 (m), 1446 (m), 1321 (m), 1295 (w), 1219 (m), 1180 (w), 991 (w), 793 (w), 731 (m), $682(\mathrm{w}), 649(\mathrm{w}) \mathrm{cm}^{-1}$. GC-MS (EI, 70eV); m/z (\%): 41 (8), 55 (6), 65 (13), 67 15
Formatted: Font: Not Italic

Deleted: $1.58\left(\mathrm{~m}, 6 \mathrm{H}, \mathrm{CH}_{2}(\mathrm{Ad})\right)$, 
(9), 77 (8), 79 (24), 81 (7), 91 (7), 92 (18), 93 (23), 107 (12), 120 (20), 135 (100), 136

(11), $227(6), 255\left(\mathrm{M}^{+}, 24\right), 256(5)$.

4.3.3. 2-(1-Adamantyl)-1-(3-aminophenyl)ethanone (8) was purified by column

chromatography ( silica gel, system b) to yield $289 \mathrm{mg}(92 \%)$ of a pale orange

crystalline powder. Mp $66-68{ }^{\circ} \mathrm{C}, \mathrm{R}_{\mathrm{f}} 0.25$ (system c), anal. calcd for $\mathrm{C}_{18} \mathrm{H}_{23} \mathrm{NO}$ : $\mathrm{C}$,

80.26\%; H, 8.61\%; N, 5.20\%; found C, 80.11\%; H, 8.49\%; N, 5.23\%. ${ }^{1} \mathrm{H}$ NMR

$\left(\mathrm{CDCl}_{3}\right): \delta 1.65\left(\mathrm{~m}, 12 \mathrm{H}, \mathrm{CH}_{2}(\mathrm{Ad})\right), 1.95(\mathrm{~m}, 3 \mathrm{H}, \mathrm{CH}(\mathrm{Ad})), 2.67$ (s, 2H, $\left.\mathrm{CH}_{2} \mathrm{CO}\right)$,

$3.80\left(\mathrm{~s}, 2 \mathrm{H}, \mathrm{NH}_{2}\right), 6.86(\mathrm{~d}, 1 \mathrm{H}, J=6.9 \mathrm{~Hz}, \mathrm{Ph}), 7.20-7.33(\mathrm{~m}, 3 \mathrm{H}, \mathrm{Ph}) \mathrm{ppm} .{ }^{13} \mathrm{C} \mathrm{NMR}$

$\left(\mathrm{CDCl}_{3}\right): \delta 29.0(\mathrm{CH}), 34.1(\mathrm{C}), 37.0\left(\mathrm{CH}_{2}\right), 43.2\left(\mathrm{CH}_{2}\right), 51.5\left(\mathrm{CH}_{2}\right), 114.3(\mathrm{CH})$

$119.3(\mathrm{CH}), 119.5(\mathrm{CH}), 129.4(\mathrm{CH}), 140.4(\mathrm{C}), 146.8(\mathrm{C}), 200.7(\mathrm{CO}) \mathrm{ppm}$.

IR (KBr): 3459 (m), 3405 (m), 3328 (m), 2899 (s), 2846 (s), 1660 (s), 1627 (m), 1595

(m), $1453(\mathrm{~m}), 1326(\mathrm{~m}), 1287(\mathrm{~m}), 1197(\mathrm{w}), 1162(\mathrm{w}), 1143(\mathrm{w}), 1096(\mathrm{w}), 991(\mathrm{w})$,

$903(\mathrm{w}), 884(\mathrm{w}), 777(\mathrm{w}), 691(\mathrm{w}), 677(\mathrm{w}) \mathrm{cm}^{-1}$. GC-MS (EI, 70eV); m/z (\%): 41

(8), 55 (5), 65 (18), 77 (7), 79 (12), 91 (10), 92 (42), 93 (19), 106 (6), 107 (10), 120

(100), 121 (14), 135 (20), 241 (5), 251 (6), $269\left(\mathrm{M}^{+}, 51\right), 270$ (10).

\subsection{General procedure for nitro ketones reduction to nitro alcohols 9-11}

The corresponding ketone $(0.84 \mathrm{mmol})$ was dissolved in warm ethanol $(5 \mathrm{~mL})$ and solution was cooled in ice bath. A small portion of starting material formed a soft precipitate. The sodium borohydride $(40 \mathrm{mg}, 1.04 \mathrm{mmol})$ was added into this dispersion in one portion at $0{ }^{\circ} \mathrm{C}$. The reaction mixture was vigorously stirred and temperature was allowed to reach $20^{\circ} \mathrm{C}$. After the consumption of all starting

material (according to TLC), the mixture was poured onto $1 \mathrm{M} \mathrm{HCl}(10 \mathrm{~mL})$ and a pale

Deleted: into yellow solid precipitated. Mixture was extracted twice with diethyl ether (10 mL),

16 
collected organic portions were washed with brine and dried over $\mathrm{Na}_{2} \mathrm{SO}_{4}$. Crude product was obtained after removing of the solvent in vacuo.

4.4.1. 1-Adamantyl-(4-nitrophenyl)methanol (9) was purified by crystallization from methanol to yield $233 \mathrm{mg}$ (96\%) of a pale yellow crystals. Mp 191-192 ${ }^{\circ} \mathrm{C}, \mathrm{R}_{\mathrm{f}} 0.11$ (system b), anal. calcd for $\mathrm{C}_{17} \mathrm{H}_{21} \mathrm{NO}_{3}$ : C, 71.06\%; $\mathrm{H}, 7.37 \%$;, $4.87 \%$; O, 16.70; found $\mathrm{C}, 71.34 \% ; \mathrm{H}, 7.45 \% ; \mathrm{N}, 4.98 \% .{ }^{1} \mathrm{H} \mathrm{NMR}\left(\mathrm{CDCl}_{3}\right): \delta 1.47-1.72(\mathrm{~m}, 12 \mathrm{H}$, $\left.\mathrm{CH}_{2}(\mathrm{Ad})\right), 2.01$ (m, 4H, CH(Ad), $\left.\mathrm{OH}\right), 4.33$ (s, 1H, CHOH), 7.44 (d, J=8.1 Hz, 2H, $\mathrm{Ph}), 8.18(\mathrm{~d}, J=8.5 \mathrm{~Hz}, 2 \mathrm{H}, \mathrm{Ph}) \mathrm{ppm} .{ }^{13} \mathrm{C} \mathrm{NMR}\left(\mathrm{CDCl}_{3}\right): \delta 28.4(\mathrm{CH}), 37.1\left(\mathrm{CH}_{2}\right)$, $37.7(\mathrm{C}), 38.3\left(\mathrm{CH}_{2}\right), 82.3(\mathrm{CH}), 122.8(\mathrm{CH}), 128.8(\mathrm{CH}), 147.5(\mathrm{C}), 148.8(\mathrm{C}) \mathrm{ppm}$. IR (KBr): 3565 (s), 3112 (w), 3081 (w), 2908 (s), 2848 (s), 1600 (m), 1506 (s), 1450 (w), $1346(\mathrm{~s}), 1312(\mathrm{~m}), 1216(\mathrm{w}), 1168(\mathrm{w}), 1105(\mathrm{~m}), 1038(\mathrm{~m}), 977$ (w), $938(\mathrm{w})$, $855(\mathrm{~m}), 830(\mathrm{w}), 800(\mathrm{w}), 760(\mathrm{w}), 720(\mathrm{~s}), 701(\mathrm{w}), 637(\mathrm{w}), 740(\mathrm{~m}) \mathrm{cm}^{-1}$. GC-MS (EI, 70eV); m/z (\%): $41(8), 44(14), 55$ (6), 67 (8), 77 (8), 79 (18), 91 (5), 93 (17), 107 (10), 121 (6), 122 (14), 135 (100), $136(12), 287\left(\mathrm{M}^{+}, 4\right)$.

\subsubsection{1-Adamantyl-(3-nitrophenyl)methanol (10) was purified by column}

chromatography (silica gel, system b) to yield $234 \mathrm{mg}$ (97\%) of a colourless

crystalline powder. Mp $104-106{ }^{\circ} \mathrm{C}, \mathrm{R}_{\mathrm{f}} 0.20$ (system b), anal. calcd for $\mathrm{C}_{17} \mathrm{H}_{21} \mathrm{NO}_{3}$ : C, 71.06\%; H, 7.37\%; N, 4.87\%; found C, 70.83\%; H, 7.18\%; N, 4.55\%. ${ }^{1} \mathrm{H}$ NMR $\left(\mathrm{CDCl}_{3}\right): \delta 1.27-1.71\left(\mathrm{~m}, 12 \mathrm{H}, \mathrm{CH}_{2}(\mathrm{Ad})\right), 1.99(\mathrm{~m}, 3 \mathrm{H}, \mathrm{CH}(\mathrm{Ad})), 2.10(\mathrm{~s}, 1 \mathrm{H}, \mathrm{OH})$, 4.33 (s, 1H, CHOH), 7.49 (m, 1H, Ph), 7.59 (m, 1H, Ph), 8.12 (m, 2H, Ph) ppm. ${ }^{13} \mathrm{C} \mathrm{NMR}\left(\mathrm{CDCl}_{3}\right): \delta 28.9(\mathrm{CH}), 32.9(\mathrm{C}), 37.2\left(\mathrm{CH}_{2}\right), 43.3\left(\mathrm{CH}_{2}\right), 54.7\left(\mathrm{CH}_{2}\right), 70.3$ $(\mathrm{CH}), 120.9(\mathrm{CH}), 122.4(\mathrm{CH}), 129.6(\mathrm{CH}), 132.1(\mathrm{CH}), 148.6(\mathrm{C}), 149.0(\mathrm{C}) \mathrm{ppm}$. IR (KBr): 3543 (m), 3432 (m), 3106 (w), 3092 (w), 2906 (s), 2848 (s), 1525 (s), 1475 
(w), $1448(w), 1349(\mathrm{~s}), 1312(\mathrm{w}), 1286(\mathrm{w}), 1194(\mathrm{w}), 1126(\mathrm{w}), 1088(\mathrm{w}), 1036(\mathrm{~m})$, $1021(\mathrm{~m}), 982(\mathrm{w}), 930(\mathrm{w}), 909(\mathrm{w}), 896(\mathrm{w}), 813(\mathrm{~m}), 722(\mathrm{~m}), 693(\mathrm{~m}), 661(\mathrm{w})$,

$618(w) \mathrm{cm}^{-1}$. GC-MS (EI, 70eV); m/z (\%): 41 (10), 55 (7), 67 (10), 77 (13), 78 (5), 79 (26), 81 (6), 91 (7), 93 (22), 105 (5), 107 (12), 135 (100), $136(11)$.

4.4.3. 2-(1-Adamantyl)-1-(3-nitrophenyl)ethanol (11) was purified by column chromatography (silica gel, system c) to yield $220 \mathrm{mg}(87 \%)$ of a colourless crystalline powder. $\mathrm{Mp} 68-69^{\circ} \mathrm{C}, \mathrm{R}_{\mathrm{f}} 0.51$ (system c), anal. calcd for $\mathrm{C}_{18} \mathrm{H}_{23} \mathrm{NO}_{3}$ : $\mathrm{C}$, $71.73 \%$; H, 7.69\%; N, 4.65\%; found C, 71.56\%; H, 7.44\%; N, 4.83\%. ${ }^{1} \mathrm{H}$ NMR $\left(\mathrm{CDCl}_{3}\right): \delta 1.50\left(\mathrm{~d}, J=2.6,1 \mathrm{H}, \mathrm{AdCH}^{\mathrm{A}} \mathrm{H}^{\mathrm{B}}\right), 1.59-1.79\left(\mathrm{~m}, 12 \mathrm{H}, \mathrm{CH}_{2}(\mathrm{Ad})\right), 1.88(\mathrm{~d}$, $\left.J=3.6 \mathrm{~Hz}, 1 \mathrm{H}, \mathrm{AdCH}^{\mathrm{A}} \mathbf{H}^{\mathbf{B}}\right), 2.02(\mathrm{~m}, 3 \mathrm{H}, \mathrm{CH}(\mathrm{Ad})), 5.05(\mathrm{~m}, 1 \mathrm{H}, \mathrm{PhCHOH}), 7.52(\mathrm{t}$, $J=7.9 \mathrm{~Hz}, 1 \mathrm{H}, \mathrm{Ph}), 7.70(\mathrm{~d}, J=7.6 \mathrm{~Hz}, 1 \mathrm{H}, \mathrm{Ph}), 8.13$ (d, J=8.3 Hz, 1H, Ph), 8.23 (s, 1H, Ph) ppm. ${ }^{13} \mathrm{C} \mathrm{NMR}\left(\mathrm{CDCl}_{3}\right): \delta 28.9(\mathrm{CH}), 32.9(\mathrm{C}), 37.2\left(\mathrm{CH}_{2}\right), 43.3\left(\mathrm{CH}_{2}\right), 54.7$ $\left(\mathrm{CH}_{2}\right), 70.3(\mathrm{CH}), 120.9(\mathrm{CH}), 122.4(\mathrm{CH}), 129.6(\mathrm{CH}), 132.1(\mathrm{CH}), 148.6(\mathrm{C}), 149.0$ (C) ppm. IR (KBr): 3380 (bs), 3090 (w), 3072 (w), 2899 (s), 2845 (s), 1525 (s), 1445 (m), 1349 (s), $1314(\mathrm{w}), 1199(\mathrm{w}), 1160(\mathrm{w}), 1103(\mathrm{~m}), 1066(\mathrm{~m}), 1014(\mathrm{w}), 969(\mathrm{w})$, $827(\mathrm{w}), 802(\mathrm{w}), 740(\mathrm{~m}), 722(\mathrm{~m}), 698(\mathrm{~m}), 676(\mathrm{~m}) \mathrm{cm}^{-1}$. GC-MS (EI, 70eV); $\mathrm{m} / \mathrm{z}$ $(\%):$ 41(17), 43(5), 53(5), 55(12), 65(5), 67(22), 69(7), 77(21), 78(11), 79(35), 80(5), 81(21), 91(20), 92(11), 93(43), 94(8), 95(6), 105(13), 106(8), 107(28), 121(8), 134(6), 135(100), 136(14), 149(58), 150(15), 152(22), 266(17), 283(14).

\subsection{General procedure for nitro alcohols reduction to amino alcohols 12-15}

The alcohols 13 and 14 and methoxy compound 12 were prepared from corresponding nitro alcohols in the same way as the ketones $\mathbf{6}-\mathbf{8}$.

Deleted: Compound 15 was prepared in dioxane according the same procedure as 18 
1

2

3

4

5

6

7

8

9

4.5.1. 4-[1-Adamantyl(methoxy)methyl] anilinium chloride $(\mathbf{1 2} \cdot \mathbf{H C l})$ crystallised as the hydrochloride salt directly from reaction mixture and no further purification was necessary. Yield: $275 \mathrm{mg}(85 \%)$ of a gold-yellow plate crystals. $\mathrm{Mp}>350{ }^{\circ} \mathrm{C}, \mathrm{R}_{\mathrm{f}}($ free base) 0.27 (system b), anal. calcd for $\mathrm{C}_{18} \mathrm{H}_{26} \mathrm{ClNO}$ : C, 70.22\%; $\mathrm{H}, 8.51 \%$; N, $4.55 \%$; found C, 69.94\%; H, 8.37\%; N, 4.75\%. ${ }^{1} \mathrm{H}$ NMR (DMSO- $\left.d_{6}\right): \delta 1.34-1.60(\mathrm{~m}, 12 \mathrm{H}$, $\left.\mathrm{CH}_{2}(\mathrm{Ad})\right), 1.89$ (m, 3H, CH(Ad)), 3.08 (s, 3H, $\left.\mathrm{OCH}_{3}\right), 3.71$ (s, 1H, $\mathrm{CHOCH}_{3}$ ), 7.27$7.36(\mathrm{~m}, 4 \mathrm{H}, \mathrm{Ph}), 10.28\left(\mathrm{bs}, 3 \mathrm{H}, \mathrm{NH}_{3}{ }^{+}\right) \mathrm{ppm} .{ }^{13} \mathrm{C}$ NMR (DMSO-d $): \delta 27.5(\mathrm{CH}), 36.5$ $\left(\mathrm{CH}_{2}\right), 37.7\left(\mathrm{CH}_{2}\right), 56.9\left(\mathrm{CH}_{3}\right), 90.8(\mathrm{CH}), 122.0(\mathrm{CH}), 129.3(\mathrm{CH}), 131.0(\mathrm{C}), 137.8$ (C) ppm. IR (KBr): 3437 (bs), 2905 (s), 2847 (s), 2562 (s), 1625 (m), 1578 (m), 1556 (m), 1507 (s), $1452(\mathrm{~m}), 1360(\mathrm{w}), 1347(\mathrm{w}), 1316(\mathrm{w}), 1241(\mathrm{w}), 1175(\mathrm{w}), 1133(\mathrm{w})$, 1085 (s), 997 (w), 826 (w), 529 (m) cm . GC-MS (IE, 70eV); m/z (\%): 120 (7), 121 (6), 136 (100), $137(9), 271\left(\mathrm{M}^{+}, 2\right)$.

4.5.2. 1-Adamantyl-(3-aminophenyl)methanol (13) Crude material was purified by column chromatography ( crystalline powder. $\mathrm{Mp} 137-139^{\circ} \mathrm{C}, \mathrm{R}_{\mathrm{f}} 0.11$ (system b), anal. calcd for $\mathrm{C}_{17} \mathrm{H}_{23} \mathrm{NO}$ : $\mathrm{C}$, 79.33\%; H, 9.01\%; N, 5.44\%; found C, 79.57\%; H, 9.15\%; N, 5.72\%. ${ }^{1} \mathrm{H}$ NMR $\left(\mathrm{CDCl}_{3}\right): \delta 1.49-1.66\left(\mathrm{~m}, 12 \mathrm{H}, \mathrm{CH}_{2}(\mathrm{Ad})\right), 1.97(\mathrm{~m}, 3 \mathrm{H}, \mathrm{CH}(\mathrm{Ad})), 3.62\left(\mathrm{bs}, 2 \mathrm{H}_{2} \mathrm{NH}_{2}\right)$, $4.12(\mathrm{~s}, 1 \mathrm{H}, \mathrm{CHOH}), 6.60-6.67(\mathrm{~m}, 3 \mathrm{H}, \mathrm{Ph}), 7.10(\mathrm{t}, J=7.6 \underline{\mathrm{Hz}}, 1 \mathrm{H}, \mathrm{Ph}) \mathrm{ppm} .{ }^{1} \mathrm{H}$ NMR (DMSO-d $\left.d_{6}\right): \delta 1.37-1.63\left(\mathrm{~m}, 12 \mathrm{H}, \mathrm{CH}_{2}(\mathrm{Ad})\right), 1.89(\mathrm{~m}, 3 \mathrm{H}, \mathrm{CH}(\mathrm{Ad})), 3.86(\mathrm{~d}, J=3.8$ $\mathrm{Hz}, 1 \mathrm{H}, \mathrm{CHOH}), 4.77$ (d, J=3.8 Hz, 1H, CHOH), 4.85 (s, 2H, NH $\left.\mathrm{NH}_{2}\right), 6.34-6.41$ (m, $2 \mathrm{H}, \mathrm{Ph}), 6.46(\mathrm{~s}, 1 \mathrm{H}, \mathrm{Ph}), 6.89(\mathrm{t}, J=7.6 \mathrm{~Hz}, 1 \mathrm{H}, \mathrm{Ph}) \mathrm{ppm} .{ }^{13} \mathrm{C} \mathrm{NMR}\left(\mathrm{CDCl}_{3}\right): \delta 28.6$ $(\mathrm{CH}), 29.9(\mathrm{C}), 37.3\left(\mathrm{CH}_{2}\right), 38.5\left(\mathrm{CH}_{2}\right), 83.2(\mathrm{CH}), 114.3(\mathrm{CH}), 114.8(\mathrm{CH}), 118.7$ (CH), $128.5(\mathrm{CH}), 142.8(\mathrm{C}), 145.9(\mathrm{C}) \mathrm{ppm} . \mathrm{IR}(\mathrm{KBr}): 3394(\mathrm{~m}), 2901$ (s), 2847 (m), $2359(\mathrm{w}), 1606(\mathrm{~m}), 1490(\mathrm{w}), 1457(\mathrm{~m}), 1302(\mathrm{w}), 1125(\mathrm{w}), 1036(\mathrm{~m}), 885(\mathrm{w}), 750$ 19 


\begin{abstract}
(m), $730(\mathrm{~m}), 700(\mathrm{~m}), 667$ (w), 585 (w), 418 (w) $\mathrm{cm}^{-1}$. GC-MS (EI, 70eV); m/z (\%): 41 (8), 55 (7), 65 (5), 67 (10), 77 (16), 79 (24), 81 (7), 91 (6), 92 (5), 93 (25), 94 (17), 107 (13), 120 (6), 121 (54), 122 (13), 135 (100), 136 (11), $257\left(\mathrm{M}^{+}, 20\right), 258$ (4).
\end{abstract}

4.5.3. 2-(1-Adamantyl)-1-(3-aminophenyl)ethanol (14) Crude material was purified by washing with hexane to yield $234 \mathrm{mg}(82 \%)$ of a colourless crystalline powder. Mp $142-145^{\circ} \mathrm{C}, \mathrm{R}_{\mathrm{f}} 0.57$ (system a), anal. calcd for $\mathrm{C}_{18} \mathrm{H}_{25} \mathrm{NO}$ : C, 79.66\%; H, 9.28\%; N, 5.16\%; found C, 79.73\%; $\mathrm{H}, 9.52 \% ; \mathrm{N}, 5.38 \% .{ }^{1} \mathrm{H} \mathrm{NMR}\left(\mathrm{CDCl}_{3}\right): \delta 1.50(\mathrm{~d}, J=2.6$ $\left.\mathrm{Hz}, 1 \mathrm{H}, \operatorname{AdCH}^{\mathrm{A}} \mathrm{H}^{\mathrm{B}}\right), 1.59-1.79\left(\mathrm{~m}, 12 \mathrm{H}, \mathrm{CH}_{2}(\mathrm{Ad})\right), 1.88(\mathrm{~d}, J=3.6 \mathrm{~Hz}, 1 \mathrm{H}$, $\left.\mathrm{AdCH}^{\mathrm{A}} \mathbf{H}^{\mathbf{B}}\right), 2.02(\mathrm{~m}, 3 \mathrm{H}, \mathrm{CH}(\mathrm{Ad})), 5.05(\mathrm{~m}, 1 \mathrm{H}, \mathrm{PhCHOH}), 7.52(\mathrm{t}, J=7.9 \mathrm{~Hz}, 1 \mathrm{H}$, Ph), 7.70 (d, J=7.6 Hz, 1H, Ph), 8.13 (d, J=8.3 Hz, 1H, Ph), 8.23 (s, 1H, Ph) ppm. ${ }^{13} \mathrm{C} \mathrm{NMR}\left(\mathrm{CDCl}_{3}\right): \delta 28.9(\mathrm{CH}), 32.9(\mathrm{C}), 37.2\left(\mathrm{CH}_{2}\right), 43.3\left(\mathrm{CH}_{2}\right), 54.7\left(\mathrm{CH}_{2}\right), 70.3$ (CH), $120.9(\mathrm{CH}), 122.4(\mathrm{CH}), 129.6(\mathrm{CH}), 132.1(\mathrm{CH}), 148.6(\mathrm{C}), 149.0(\mathrm{C}) \mathrm{ppm}$. IR (KBr): 3380 (bs), 3090 (w), 3072 (w), 2899 (s), 2845 (s), 1525 (s), 1445 (m), 1349 (s), $1314(w), 1199(w), 1160(w), 1103(m), 1066(m), 1014(w), 969(w), 827(w)$, $802(\mathrm{w}), 740(\mathrm{~m}), 722(\mathrm{~m}), 698(\mathrm{~m}), 676(\mathrm{~m}) \mathrm{cm}^{-1}$. GC-MS (EI, 70eV); m/z $(\%): 41$ (8), 55 (5), 65 (5), 67 (7), 77 (17), 79 (11), 81 (5), 91 (7), 92 (5), 93 (15), 94 (72), 95 (7), $107(6), 120(6), 121(20), 122(100), 123(9), 135(7), 253(5), 271\left(\mathrm{M}^{+}, 28\right), 272$ (6).

\subsubsection{1-Adamantyl-(4-aminophenyl)methanol (15) The nitroalcohol 9 (259 mg, 0.90 mmol) was dissolved in ethanol (40 mL) under $\mathrm{H}_{2}$ atmosphere and large excess of Ra- $\underline{\text { Ni was added portionwise until starting material disappeared. Ra-Ni was filtered off, }}$ the filtrate was diluted with water and extracted several times with diethyl ether.

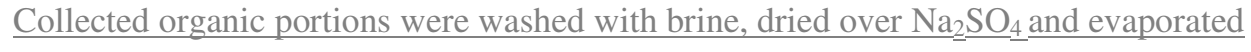
20 
1

2

3

4

5

6

7

8

9

10

11

12

13

14

15

16

17

18

19

20

21

22

23

24

25

26

27

28

29

30

31

32

33

34

35

36

37

38

39

40

41

42

43

44

45

46

47

48

49

50

51

52

53

54

55

56

57

58

59

60

in vacuo. Crude material was purified by column chromatography (silica gel, system a) to yield $227 \mathrm{mg}(98 \%)$ of a pale yellow crystalline powder. $\mathrm{Mp} 143-146{ }^{\circ} \mathrm{C}, \mathrm{R}_{\mathrm{f}}$ 0.41 (system a), anal. calcd for $\mathrm{C}_{17} \mathrm{H}_{23} \mathrm{NO}$ : C, 79.33\%; H, 9.01\%; N, 5.44\%; found C, 79.09\%; H, 9.23\%; N, 5.28\%. ${ }^{1} \mathrm{H} \mathrm{NMR}\left(\mathrm{CDCl}_{3}\right): \delta 1.48-1.67$ (m, 12H, $\left.\mathrm{CH}_{2}(\mathrm{Ad})\right)$, 1.97 (m, 3H, CH(Ad)), 3.53 (bs, 2 $\mathrm{H}_{2} \mathrm{NH}_{2}$ ), 4.11 (s, 1H, CHOH), 6.64 (d, J=8.3 Hz, 2H, Ph), $7.06(\mathrm{~d}, J=8.1 \mathrm{~Hz}, 2 \mathrm{H}, \mathrm{Ph}) \mathrm{ppm} .{ }^{13} \mathrm{C} \mathrm{NMR}\left(\mathrm{CDCl}_{3}\right): \delta 28.8(\mathrm{CH}), 29.9(\mathrm{C})$, $37.5\left(\mathrm{CH}_{2}\right), 38.5\left(\mathrm{CH}_{2}\right), 83.1(\mathrm{CH}), 114.5(\mathrm{CH}), 128.9(\mathrm{CH}), 131.8(\mathrm{C}), 145.8(\mathrm{C})$ ppm. IR (KBr): 3378 (m), 2905 (s), 2847 (m), 1615 (m), 1514 (m), 1447 (w), 1265 (m), $1175(\mathrm{w}), 1128(\mathrm{w}), 1046(\mathrm{~m}), 844(\mathrm{w}), 811(\mathrm{w}), 572(\mathrm{~m}), 535(\mathrm{w}), 481(\mathrm{w}) \mathrm{cm}^{-1}$. GC-MS (EI, 70eV); m/z (\%): 77 (9), 79 (7), 93 (7), 94 (12), 120 (9), 121 (38), 122 (100), $123(8), 135(5), 257\left(\mathrm{M}^{+}, 4\right)$.

\subsection{General procedures for nitrodithianes 16-18 and nitrodithiolanes 19, 20}

\section{formation}

The ketone $(0.35 \mathrm{mmol})$ was dissolved in dichloromethane $(2 \mathrm{~mL})$ and corresponding dithiol (1,2-ethanedithiol or 1,3-propanedithiol; $0.55 \mathrm{mmol})$ was added. The solution was cooled in ice bath to $0{ }^{\circ} \mathrm{C}$ and stirred for $30 \mathrm{~min}$. After this period, boron trifluoride-diethyl ether $(1.00 \mathrm{mmol})$ was added dropwise and the mixture was stirred at room temperature until TLC indicated complete disappearing of the starting material. The mixture was diluted with $\mathrm{CH}_{2} \mathrm{Cl}_{2}(20 \mathrm{~mL})$ and washed three times with $5 \%$ solution of $\mathrm{NaOH}(10 \mathrm{~mL})$. The organic layer was washed twice with brine, dried over $\mathrm{Na}_{2} \mathrm{SO}_{4}$ and evaporated in vacuo.

4.6.1. 2-(1-Adamantyl)-2-(3-nitrophenyl)-1,3-dithiane (16) was purified by crystallisation from hexane/ $\mathrm{CH}_{2} \mathrm{Cl}_{2}$ to yield $121 \mathrm{mg}$ (92\%) of yellow needles. 
Mp 191-193 ${ }^{\circ} \mathrm{C}, \mathrm{R}_{\mathrm{f}} 0.42$ (system c), anal. calcd for $\mathrm{C}_{20} \mathrm{H}_{25} \mathrm{NO}_{2} \mathrm{~S}_{2}$ : C, 63.96\%; $\mathrm{H}$, $6.71 \%$; N, 3.73\%; S, 17.08\%; found C, 64.23\%; H, 6.55\%; N, 3.37\%; S, 17.12\%. ${ }^{1} \mathrm{H}$ NMR $\left(\mathrm{CDCl}_{3}\right): \delta 1.58\left(\mathrm{~m}, 6 \mathrm{H}, \mathrm{CH}_{2}(\mathrm{Ad})\right), 1.83\left(\mathrm{~m}, 8 \mathrm{H}, \mathrm{CH}_{2}(\mathrm{Ad}), \mathrm{SCH}_{2} \mathrm{CH}_{2}\right), 1.97$ (m, 3H, CH(Ad)), $2.45\left(\mathrm{~m}, 2 \mathrm{H}, \mathrm{SCH}^{\mathrm{A}} \mathrm{H}^{\mathrm{B}}\right), 2.67\left(\mathrm{~m}, 1 \mathrm{H}, \mathrm{SCH}^{\mathrm{C}}\right), 2.71\left(\mathrm{~m}, 1 \mathrm{H}, \mathrm{SCH}^{\mathrm{D}}\right)$, 7.57 (t, J=7.9 Hz, 1H, Ph), 8.15 (d, J=8.3 Hz, 1H, Ph), 8.33 (d, J=7.9 Hz, 1H, Ph), $8.85(\mathrm{~s}, 1 \mathrm{H}, \mathrm{Ph}) \mathrm{ppm} .{ }^{13} \mathrm{C} \mathrm{NMR}\left(\mathrm{CDCl}_{3}\right): \delta 25.4\left(\mathrm{CH}_{2}\right), 27.8\left(\mathrm{CH}_{2}\right), 28.9(\mathrm{CH}), 36.8$ $\left(\mathrm{CH}_{2}\right), 37.6\left(\mathrm{CH}_{2}\right), 41.7(\mathrm{C}), 70.2(\mathrm{C}), 122.0(\mathrm{CH}), 127.4(\mathrm{CH}), 128.7(\mathrm{CH}), 138.6$ (CH), 141.0 (C), 148.7 (C) ppm. IR (KBr): 2904 (s), 2846 (s), 1521 (s), 1469 (w), $1447(w), 1420(w), 1351(\mathrm{~s}), 1310(\mathrm{w}), 1273(\mathrm{w}), 1172(\mathrm{w}), 1093(\mathrm{w}), 1010(\mathrm{w}), 979$ (w), $940(w), 892(w), 813(w), 787(w) 726(m), 694(m) \mathrm{cm}^{-1}$. GC-MS (EI, 70eV); $\mathrm{m} / \mathrm{z}(\%): 41(11), 55(5), 67(8), 77(6), 79(20), 81(5), 91(6), 93$ (17), $107(10), 120$ Deleted: $\mathrm{m} / \mathrm{z}$ (5), 135 (100), 136 (12), 224 (43), 225 (6), $240(10), 375\left(\mathrm{M}^{+}, 3\right)$.

4.6.2. 2-(1-Adamantyl)-2-(4-nitrophenyl)-1,3-dithiane (17) was purified by crystallisation from hexane/ $\mathrm{CH}_{2} \mathrm{Cl}_{2}$ to yield $120 \mathrm{mg}$ (91\%) of yellow needles. Mp 218-220 ${ }^{\circ} \mathrm{C}, \mathrm{R}_{\mathrm{f}} 0.51$ (system b), anal. calcd for $\mathrm{C}_{20} \mathrm{H}_{25} \mathrm{NO}_{2} \mathrm{~S}_{2}: \mathrm{C}, 63.96 \% ; \mathrm{H}$, $6.71 \%$; N, 3.73\%; S, 17.08\%; found C, 63.84\%; H, 6.52\%; N, 3.46\%; S, $16.95 \%$. ${ }^{1} \mathrm{H}$ NMR $\left(\mathrm{CDCl}_{3}\right): \delta 1.57$ (m, 6H, $\left.\mathrm{CH}_{2}(\mathrm{Ad})\right), 1.83\left(\mathrm{~m}, 8 \mathrm{H}, \mathrm{CH}_{2}(\mathrm{Ad}), \mathrm{SCH}_{2} \mathrm{CH}_{2}\right), 1.97$ (m, 3H, CH(Ad)), $2.46\left(\mathrm{~m}, 2 \mathrm{H}, \mathrm{SCH}^{\mathrm{A}} \mathrm{H}^{\mathrm{B}}\right), 2.67\left(\mathrm{~m}, 1 \mathrm{H}, \mathrm{SCH}^{\mathrm{C}}\right), 2.71\left(\mathrm{~m}, 1 \mathrm{H}, \mathrm{SCH}^{\mathrm{D}}\right)$, $8.20(\mathrm{~m}, 4 \mathrm{H}, \mathrm{Ph}) \mathrm{ppm} .{ }^{13} \mathrm{C}$ NMR $\left(\mathrm{CDCl}_{3}\right): \delta 25.3\left(\mathrm{CH}_{2}\right), 27.8\left(\mathrm{CH}_{2}\right), 28.9(\mathrm{CH}), 36.8$ $\left(\mathrm{CH}_{2}\right), 37.6\left(\mathrm{CH}_{2}\right), 41.7(\mathrm{C}), 70.4(\mathrm{C}), 122.9(\mathrm{CH}), 133.6(\mathrm{CH}), 146.1(\mathrm{C}), 146.6(\mathrm{C})$ ppm. IR (KBr): 2908 (s), 2848 (s), 1598 (m), 1515 (s), 1447 (w), 1413 (w), 1349 (s), $1308(\mathrm{~s}), 1283(\mathrm{w}), 1264(\mathrm{w}), 1110(\mathrm{~m}), 1066(\mathrm{w}), 1011(\mathrm{w}), 976(\mathrm{w}), 930(\mathrm{w}), 855$ (m), 839 (m), 794 (w), 727 (m), 696 (w) cm-1. GC-MS (IE, 70eV); m/z (\%): 41 (9), 77 
1

2

3

4

5

6

7

8

9

10

(5), 79 (17), 81 (5), 91 (5), 93 (14), 107 (8), 135 (100), 136 (12), 210 (5), 224 (21), $240(5), 375\left(\mathrm{M}^{+}, 4\right)$.

4.6.3. 2-(1-Adamantylmethyl)-2-(3-nitrophenyl)-1,3-dithiane (18) was purified by crystallisation from hexane to yield $125 \mathrm{mg}$ (92\%) of yellow needles. Mp 174$176{ }^{\circ} \mathrm{C}, \mathrm{R}_{\mathrm{f}} 0.60$ (system b), anal. calcd for $\mathrm{C}_{21} \mathrm{H}_{27} \mathrm{NO}_{2} \mathrm{~S}_{2}: \mathrm{C}, 64.74 \% ; \mathrm{H}, 6.99 \% ; \mathrm{N}$, $3.60 \%$; S, $16.46 \%$; found C, $64.58 \%$; H, 7.17\%; N, 3.87\%; S, 16.72\%. ${ }^{1} \mathrm{H}$ NMR $\left(\mathrm{CDCl}_{3}\right): \delta 1.36\left(\mathrm{~m}, 6 \mathrm{H}, \mathrm{CH}_{2}(\mathrm{Ad})\right), 1.51\left(\mathrm{~m}, 6 \mathrm{H}, \mathrm{CH}_{2}(\mathrm{Ad})\right), 1.78$ (m, 3H, $\left.\mathrm{CH}(\mathrm{Ad})\right)$, $1.94\left(\mathrm{~m}, 2 \mathrm{H}, \mathrm{SCH}_{2} \mathrm{CH}_{2}\right), 2.04$ (s, 2H, AdCH $\left.\mathbf{H}_{2}\right), 2.64$ (m, 4H, SCH $), 7.54$ (t, J=7.9 Hz, 1H, Ph), 8.13 (d, J=7.9 Hz, 1H, Ph), 8.33 (d, J=9.2 Hz, 1H, Ph), 8.86 (s, 1H, Ph) ppm. ${ }^{13} \mathrm{C}$ NMR $\left(\mathrm{CDCl}_{3}\right): \delta 24.8\left(\mathrm{CH}_{2}\right), 28.1(\mathrm{CH}), 28.9(\mathrm{CH}), 36.1(\mathrm{C}), 36.8\left(\mathrm{CH}_{2}\right), 44.0$ $\left(\mathrm{CH}_{2}\right), 57.6(\mathrm{C}), 59.8\left(\mathrm{CH}_{2}\right), 122.2(\mathrm{CH}), 125.1(\mathrm{CH}), 129.3(\mathrm{CH}), 136.0(\mathrm{CH}), 145.7$ (C), 148.7 (C) ppm. IR (KBr): 3083 (w), 2899 (s), 2845 (s), 2672 (w), 1573 (w), 1523 (s), $1470(\mathrm{w}), 1448(\mathrm{w}), 1422(\mathrm{~m}), 1350(\mathrm{~s}), 1311(\mathrm{~m}), 1281(\mathrm{w}), 1270(\mathrm{~m}), 1101(\mathrm{~m})$, $1091($ w), $1079($ w), 1032 (w), $996($ w), 863 (w), 802 (m), 776 (w), $733($ m), 707 (w), 687 (m) $\mathrm{cm}^{-1}$. GC-MS (EI, 70eV); m/z (\%): 41 (11), 55 (11), 67 (13), 69 (5), 77 (8), 79 (28), 81 (10), 91 (10), 93 (24), 107 (18), 135 (100), 136 (13), 239 (43), 315 (7), $389\left(\mathrm{M}^{+}, 6\right)$.

4.6.4. 2-(1-Adamantyl)-2-(4-nitrophenyl)-1,3-dithiolane (19) was purified by crystallisation from hexane/ $\mathrm{CH}_{2} \mathrm{Cl}_{2}$ to yield $115 \mathrm{mg}$ (91\%) of colourless needles. Mp $185-189{ }^{\circ} \mathrm{C}, \mathrm{R}_{\mathrm{f}} 0.51$ (system b), anal. calcd for $\mathrm{C}_{19} \mathrm{H}_{23} \mathrm{NO}_{2} \mathrm{~S}_{2}$ : C, 63.12\%; $\mathrm{H}, 6.41 \%$; N, 3.87\%; S, 17.74\%; found C, 63.41\%; H, 6.51\%; N, 4.12\%; S, $17.45 \% .{ }^{1} \mathrm{H}$ NMR $\left(\mathrm{CDCl}_{3}\right): \delta 1.57\left(\mathrm{~m}, 6 \mathrm{H}, \mathrm{CH}_{2}(\mathrm{Ad})\right), 1.79\left(\mathrm{~m}, 6 \mathrm{H}, \mathrm{CH}_{2}(\mathrm{Ad})\right), 1.99$ (m, 3H, $\left.\mathrm{CH}(\mathrm{Ad})\right)$, $2.98\left(\mathrm{~m}, 2 \mathrm{H}, \mathrm{SCH}^{\mathrm{A}} \mathrm{H}^{\mathrm{B}}\right), 3.27\left(\mathrm{~m}, 2 \mathrm{H}, \mathrm{SCH}^{\mathrm{A}} \mathbf{H}^{\mathbf{B}}\right), 7.97(\mathrm{~m}, 2 \mathrm{H}, \mathrm{Ph}), 8.10(\mathrm{~m}, 2 \mathrm{H}, \mathrm{Ph})$ 
ppm. ${ }^{13} \mathrm{C} \mathrm{NMR}\left(\mathrm{CDCl}_{3}\right): \delta 29.0(\mathrm{CH}), 36.6\left(\mathrm{CH}_{2}\right), 38.8\left(\mathrm{CH}_{2}\right), 39.9\left(\mathrm{CH}_{2}\right), 41.3(\mathrm{C})$, $86.4(\mathrm{C}), 121.6(\mathrm{CH}), 132.0(\mathrm{CH}), 146.8(\mathrm{C}), 151.6(\mathrm{C}) \mathrm{ppm}$. IR (KBr): $2903(\mathrm{~s})$, $2845(\mathrm{~m}), 1600(\mathrm{~m}), 1515(\mathrm{~s}), 1447$ (w), $1400(\mathrm{w}), 1345$ (s), 1308 (w), $1145(\mathrm{w}), 1110$ (m), $1014(\mathrm{w}), 979(\mathrm{~m}), 853(\mathrm{~m}), 842(\mathrm{~m}), 809(\mathrm{w}), 728(\mathrm{~m}), 697(\mathrm{~m}), 638(\mathrm{w}), 503$ (w) $\mathrm{cm}^{-1}$. GC-MS (EI, 70eV); m/z (\%): 41 (6), 67 (7), 77 (5), 79 (18), 81 (5), 91 (5), 93 (17), 107 (10), 135 (100), 136 (13), 196 (10), 210 (8), 361(M+1 3).

4.6.5. 2-(1-Adamantylmethyl)-2-(3-nitrophenyl)-1,3-dithiolane (20) was purified by crystallisation from hexane/ $\mathrm{CH}_{2} \mathrm{Cl}_{2}$ to yield $110 \mathrm{mg}(84 \%)$ of a pale yellow crystals. Mp 142-148 ${ }^{\circ} \mathrm{C}, \mathrm{R}_{\mathrm{f}} 0.51$ (system c), anal. calcd for $\mathrm{C}_{20} \mathrm{H}_{25} \mathrm{NO}_{2} \mathrm{~S}_{2}$ : C, $63.96 \% ; \mathrm{H}$, $6.71 \%$; N, 3.73\%; S, 17.08\%; found C, 63.98\%; H, 6.58\%; N, 3.95\%; S, $16.82 \%$. ${ }^{1} \mathrm{H} \mathrm{NMR}\left(\mathrm{CDCl}_{3}\right): \delta 1.30\left(\mathrm{~m}, 6 \mathrm{H}, \mathrm{CH}_{2}(\mathrm{Ad})\right), 1.51\left(\mathrm{~m}, 6 \mathrm{H}, \mathrm{CH}_{2}(\mathrm{Ad})\right), 1.80(\mathrm{~m}, 3 \mathrm{H}$, $\mathrm{CH}(\mathrm{Ad})), 2.52$ (s, 2H, CCH $\left.\mathbf{H}_{2} \mathrm{Ad}\right), 3.06\left(\mathrm{~m}, 2 \mathrm{H}, \mathrm{SCH}^{\mathrm{A}} \mathrm{H}^{\mathrm{B}}\right), 3.38\left(\mathrm{~m}, 2 \mathrm{H}, \mathrm{SCH}^{\mathrm{A}} \mathbf{H}^{\mathbf{B}}\right)$, 7.46 (t, J=7.9 Hz, 1H, Ph), 8.07 (d, J=7.9 Hz, 1H, Ph), 8.15 (d, J=7.9 Hz, 1H, Ph), $8.71(\mathrm{~s}, 1 \mathrm{H}, \mathrm{Ph}) \mathrm{ppm} .{ }^{13} \mathrm{C} \mathrm{NMR}\left(\mathrm{CDCl}_{3}\right): \delta 28.8(\mathrm{CH}), 35.5(\mathrm{C}), 36.8\left(\mathrm{CH}_{2}\right), 38.9$ $\left(\mathrm{CH}_{2}\right), 43.6\left(\mathrm{CH}_{2}\right), 58.3\left(\mathrm{CH}_{2}\right), 72.5(\mathrm{C}), 122.2(\mathrm{CH}), 123.0(\mathrm{CH}), 128.7(\mathrm{CH}), 134.1$ (CH), 148.0 (C), 149.1 (C) ppm. IR (KBr): 3077 (w), 2921 (s), 2902 (s), 2888 (s), 2841 (s), $1524(\mathrm{~s}), 1447$ (w), $1348(\mathrm{~s}), 1314(\mathrm{w}), 1277$ (w), $1100(\mathrm{w}), 898(\mathrm{w}), 804$

(w), 736 (m), 685 (m), 588 (w) cm ${ }^{-1}$. GC-MS (EI, 70eV); m/z (\%): 41 (8), 55 (8), 67 Deleted: $\mathrm{m} / \mathrm{z}$ (9), 79 (23), 93 (18), 107 (9), 135 (35), 149 (6), 180 (6), 226 (100), 227 (12), 228 (10), 375( $\left.\mathrm{M}^{+}, 2\right)$.

\subsection{General procedure for preparation of aminodithianes 21 and 22}

The corresponding nitrodithiane $(0.44 \mathrm{mmol})$ was dissolved in dioxane $(7 \mathrm{~mL})$ and a suspension of Ra-Ni in hexane was added to this solution. The mixture was stirred 
1

2

3

4

5

6

7

8

9

10

11

12

13

14

15

16

17

18

19

20

21

22

23

24

25

26

27

28

29

30

31

32

33

34

35

36

37

38

39

40

41

42

43

44

45

46

47

48

49

50

51

52

53

54

55

56

57

58

59

60

and refluxed under hydrogen atmosphere. Further portions of Ra-Ni were added until TLC indicated complete disappearing of the starting material. The Ra-Ni was filtrated off, resulting solution was diluted with water $(14 \mathrm{~mL})$ and extracted with diethyl ether $(6 \times 15 \mathrm{~mL})$ and hexane $(1 \times 20 \mathrm{~mL})$. Collected organic portions were washed with water $(3 \times 30 \mathrm{~mL})$, brine $(3 \times 15 \mathrm{~mL})$ and dried over $\mathrm{Na}_{2} \mathrm{SO}_{4}$.

\subsubsection{2-(1-Adamantyl)-2-(4-aminophenyl)-1,3-dithiane hydrochloride $(\mathbf{2 1} \cdot \mathbf{H C l})$ was}

precipitated from hexane solution of crude $\mathbf{2 1}$ by introducing of dry $\mathrm{HCl}$. Yield: 146 $\mathrm{mg}(87 \%)$ of a colourless crystalline powder. Mp $140-145{ }^{\circ} \mathrm{C}, \mathrm{R}_{\mathrm{f}}$ (free base) 0.24

(system b), anal. calcd for $\mathrm{C}_{20} \mathrm{H}_{28} \mathrm{ClNS}_{2}$ : C, 62.88\%; H, 7.39\%; N, 3.67\%; S, 16.79\%; found C, $62.73 \%$; $\mathrm{H}, 7.19 \%$; N, 3.62\%; S, $16.98 \% .{ }^{1} \mathrm{H} \mathrm{NMR}\left(\mathrm{CDCl}_{3}\right): \delta 1.42-1.60$ (m, 8H, $\mathrm{CH}_{2}(\mathrm{Ad})+\mathrm{CH}_{2}$ (dithiane)), $1.75\left(\mathrm{~m}, 6 \mathrm{H}, \mathrm{CH}_{2}(\mathrm{Ad})\right), 1.92$ (m, 3H, $\mathrm{CH}(\mathrm{Ad})$ ), $2.34\left(\mathrm{~m}, 2 \mathrm{H}, \mathrm{CH}_{2}\right.$ (dithiane)), $2.72\left(\mathrm{~m}, 2 \mathrm{H}, \mathrm{CH}_{2}\right.$ (dithiane)), $7.43(\mathrm{~d}, J=8.6 \mathrm{~Hz}, 2 \mathrm{H}, \mathrm{Ph}$ ), $7.92(\mathrm{~d}, J=8.6 \mathrm{~Hz}, 2 \mathrm{H}, \mathrm{Ph}), 10.34\left(\mathrm{bs}, 3 \mathrm{H}, \mathrm{NH}_{3}\right) \mathrm{ppm} .{ }^{13} \mathrm{C} \mathrm{NMR}\left(\mathrm{CDCl}_{3}\right): \delta 24.7$ Deleted: $\mathrm{NH}_{2}$ $\left(\mathrm{CH}_{2}\right), 27.0\left(\mathrm{CH}_{2}\right), 28.0(\mathrm{CH}), 36.3\left(\mathrm{CH}_{2}\right), 37.0\left(\mathrm{CH}_{2}\right), 41.6(\mathrm{C}), 69.8(\mathrm{C}), 122.4(\mathrm{CH})$, $130.8(\mathrm{C}), 133.1(\mathrm{CH}), 136.5(\mathrm{C}) \mathrm{ppm} . \mathrm{IR}(\mathrm{KBr}): 3466$ (bs), 2905 (s), 2848 (s), 2597 (m), $1614(\mathrm{w}), 1541(\mathrm{~s}), 1504(\mathrm{~m}), 1447(\mathrm{w}), 1417(\mathrm{w}), 1359(\mathrm{w}), 1344(\mathrm{w}), 1306(\mathrm{w})$, $1279(\mathrm{w}), 1024$ (w), 978 (w), 836 (w), 789 (w), 526 (w) cm $\mathrm{cm}^{-1}$. GC-MS (EI, 70eV); m/z (\%): 41 (7), 77 (5), 79 (9), 91 (5), 93 (7), 106 (10), 120 (20), 135 (10), 136 (28), 210 (100), $211(13), 212(9), 345\left(\mathrm{M}^{+}, 1\right)$.

\subsubsection{2-(1-Adamantylmethyl)-2-(3-aminophenyl)-1,3-dithiane (22) was purified by} column chromatography (silica gel, system b) to yield $106 \mathrm{mg}$ (67\%) of a pale yellow crystalline powder. Mp $127-132^{\circ} \mathrm{C}, \mathrm{R}_{\mathrm{f}} 0.38$ (system b), anal. calcd for $\mathrm{C}_{21} \mathrm{H}_{29} \mathrm{NS}_{2}$ : C, 70.14\%; H, 8.13\%; N, 3.90\%; S, 17.83; found C, 70.33\%; H, 7.94\%; N, 4.25\%, S, 
18.08\%. ${ }^{1} \mathrm{H}$ NMR $\left(\mathrm{CDCl}_{3}\right): \delta 1.40-1.58\left(\mathrm{~m}, 12 \mathrm{H}, \mathrm{CH}_{2}(\mathrm{Ad})\right), 1.78$ (m, 3H, $\left.\mathrm{CH}(\mathrm{Ad})\right)$, 1.85-1.96 (m, 4H, SCH$\left.{ }_{2} \mathrm{CH}_{2}+\mathrm{CCH}_{2} \mathrm{Ad}\right), 2.57\left(\mathrm{~m}, 2 \mathrm{H}, \mathrm{SCH}^{\mathrm{A}} \mathrm{H}^{\mathrm{B}}\right), 2.78(\mathrm{~m}, 2 \mathrm{H}$, $\left.\mathrm{SCH}^{\mathrm{A}} \mathbf{H}^{\mathbf{B}}\right), 3.68\left(\mathrm{bs}, 2 \mathrm{H}, \mathrm{NH}_{2}\right), 6.58(\mathrm{~d}, J=7.9 \mathrm{~Hz}, 1 \mathrm{H}, \mathrm{Ph}), 7.14(\mathrm{t}, J=7.9 \mathrm{~Hz}, 1 \mathrm{H}, \mathrm{Ph})$, 7.37-7.39 (m, 2H, Ph) ppm. ${ }^{13} \mathrm{C}$ NMR $\left(\mathrm{CDCl}_{3}\right): \delta 25.2\left(\mathrm{CH}_{2}\right), 28.2\left(\mathrm{CH}_{2}\right), 29.0(\mathrm{CH})$, $36.0(\mathrm{C}), 37.0\left(\mathrm{CH}_{2}\right), 43.6\left(\mathrm{CH}_{2}\right), 59.6\left(\mathrm{CH}_{2}\right), 113.9(\mathrm{CH}), 116.7(\mathrm{CH}), 120.6(\mathrm{CH})$, $129.2(\mathrm{CH}), 143.3(\mathrm{C}), 146.6(\mathrm{C}) \mathrm{ppm}$. IR (KBr): 3443 (w), $3358(\mathrm{w}), 2363(\mathrm{w}), 1730$ (w), 1614 (s), 1598 (s), 1489 (m), 1471 (w), 1448 (s), 1417 (w), 1346 (m), 1313 (m), $1277(w), 1102(w), 993(w), 881(w), 781(m), 768(w), 710(w), 694(w), 667(w)$,

457 (w) $\mathrm{cm}^{-1}$. GC-MS (EI, 70eV); m/z (\%): 41 (17), 53 (5), 55 (14), 65 (7), 67 (17), 77 (13), 79 (44), 81 (13), 91 (25), 92 (5), 93 (36), 106 (7), 107 (19), 117 (10), 118 (30), 119 (7), 135 (100), 136 (30), 210 (81), 211 (11), 212 (8), 224 (6), 251 (5), 252 (13), 253 (7), 284 (10), 285 (70), $286(15), 359\left(\mathrm{M}^{+}, 26\right), 360(7)$.

\subsection{General procedure for preparation of amino dithiolanes 23 and 24}

The corresponding nitrodithiolane $(3.37 \mathrm{mmol})$ was dissolved in $i \mathrm{PrOH}(125 \mathrm{~mL})$ and hydrochloric acid/water (1/1, v/v, $20 \mathrm{~mL})$ and an iron powder $(424 \mathrm{mg}, 7.59 \mathrm{mmol})$ was added. Into well stirred and refluxed mixture, further portions of an iron powder (424 mg, $7.59 \mathrm{mmol}$ ) were added until TLC indicated complete disappearing of the

starting material. The mixture was poured onto a $5 \%$ solution of $\mathrm{NaOH}(120 \mathrm{~mL})$ and extracted several times with diethyl ether. Combined organic layers were washed three times with water $(3 \times 15 \mathrm{~mL})$ and dried over $\mathrm{Na}_{2} \mathrm{SO}_{4}$ overnight. The crude product was obtained after evaporation of the solvent in vacuo.

4.8.1. 2-(1-Adamantyl)-2-(4-aminophenyl)-1,3-dithiolane (23) was purified by column chromatography (silica gel, $\mathrm{CHCl}_{3}$ ) to yield $827 \mathrm{mg}(74 \%)$ of a pale orange crystalline 26 
1

2

3

4

5

6

7

8

9

10

11

12

13

14

15

16

17

18

19

20

21

22

23

24

25

26

27

28

29

30

31

32

33

34

35

36

37

38

39

40

41

42

43

44

45

46

47

48

49

50

51

52

53

54

55

56

57

58

59

60

powder. Mp $121-124^{\circ} \mathrm{C}, \mathrm{R}_{\mathrm{f}} 0.31$ (system b), anal. calcd for $\mathrm{C}_{19} \mathrm{H}_{25} \mathrm{NS}_{2}: \mathrm{C}, 68.83 \%$; $\mathrm{H}$, 7.60\%; N, 4.22\%; S, 19.34; found C, 68.73\%; H, 7.45\%; N, 4.53\%; S, $19.02 \%$.

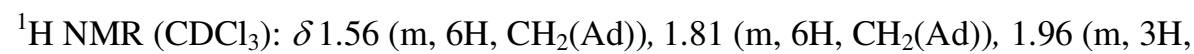
$\mathrm{CH}(\mathrm{Ad})), 2.99\left(\mathrm{~m}, 2 \mathrm{H}, \mathrm{SCH}^{\mathrm{A}} \mathrm{H}^{\mathrm{B}}\right), 3.23\left(\mathrm{~m}, 2 \mathrm{H}, \mathrm{SCH}^{\mathrm{A}} \mathbf{H}^{\mathbf{B}}\right), 3.64\left(\mathrm{~s}, 2 \mathrm{H}, \mathrm{NH}_{2}\right), 6.58(\mathrm{~d}$, $J=8.6 \mathrm{~Hz}, 2 \mathrm{H}, \mathrm{Ph}), 7.55(\mathrm{~d}, J=8.6 \mathrm{~Hz}, 2 \mathrm{H}, \mathrm{Ph}) \mathrm{ppm} .{ }^{13} \mathrm{C} \mathrm{NMR}\left(\mathrm{CDCl}_{3}\right): \delta 29.1(\mathrm{CH})$, $36.8\left(\mathrm{CH}_{2}\right), 38.4\left(\mathrm{CH}_{2}\right), 40.0\left(\mathrm{CH}_{2}\right), 41.4(\mathrm{C}), 87.3(\mathrm{C}), 113.2(\mathrm{CH}), 131.9(\mathrm{CH})$, 133.2 (C), 145.0 (C) ppm. IR (KBr): 3417 (w), 2902 (s), 2370 (m), 2341 (m), 1622 (m), 1507 (m), $1280(w), 1186(w), 977$ (w), $835(w), 652(w), 531(w) \mathrm{cm}^{-1}$. GC-MS $(\mathrm{EI}, 70 \mathrm{eV}) ; \mathrm{m} / \mathrm{z}(\%): 79(6), 93$ (5), $124(5), 136(20), 196$ (100), 197 (12), 198 (9), $331\left(\mathrm{M}^{+}, 1\right)$.

4.8.2. 2-(1-Adamantylmethyl)-2-(3-aminophenyl)-1,3-dithiolane (24) was purified by column chromatography ( silica gel, system b) to yield $1013 \mathrm{mg}(87 \%)$ of a pale yellow crystalline powder. $\mathrm{Mp} 115-120^{\circ} \mathrm{C}, \mathrm{R}_{\mathrm{f}} 0.29$ (system $\mathrm{b}$ ), anal. calcd for $\mathrm{C}_{20} \mathrm{H}_{27} \mathrm{NS}_{2}$ : C, 69.51\%; H, 7.88\%; N, 4.05\%; S, 18.56; found C, 69.64\%; H, 8.15\%; $\mathrm{N}, 4.27 \% ; \mathrm{S}, 18.33 \% .{ }^{1} \mathrm{H} \mathrm{NMR}\left(\mathrm{CDCl}_{3}\right): \delta 1.35\left(\mathrm{~m}, 6 \mathrm{H}, \mathrm{CH}_{2}(\mathrm{Ad})\right), 1.53(\mathrm{~m}, 6 \mathrm{H}$, $\left.\mathrm{CH}_{2}(\mathrm{Ad})\right), 1.80$ (s, 3H, CH(Ad)), 2.44 (s, 2H, $\left.\operatorname{AdCH}_{2} \mathrm{C}\right), 3.10\left(\mathrm{~m}, 2 \mathrm{H}, \mathrm{SCH}^{\mathrm{A}} \mathrm{H}^{\mathrm{B}}\right)$, $3.32\left(\mathrm{~m}, 2 \mathrm{H}, \mathrm{SCH}^{\mathrm{A}} \mathbf{H}^{\mathbf{B}}\right), 3.65\left(\mathrm{~s}, 2 \mathrm{H}, \mathrm{NH}_{2}\right), 6.52(\mathrm{~d}, J=7.9 \mathrm{~Hz}, 1 \mathrm{H}, \mathrm{Ph}), 7.05(\mathrm{t}, J=7.9$ $\mathrm{Hz}, 1 \mathrm{H}, \mathrm{Ph}), 7.18(\mathrm{~m}, 2 \mathrm{H}, \mathrm{Ph}) \mathrm{ppm} .{ }^{13} \mathrm{C} \mathrm{NMR}\left(\mathrm{CDCl}_{3}\right): \delta 28.9(\mathrm{CH}), 35.4(\mathrm{C}), 36.9$ $\left(\mathrm{CH}_{2}\right), 38.5\left(\mathrm{CH}_{2}\right), 43.2\left(\mathrm{CH}_{2}\right), 58.4\left(\mathrm{CH}_{2}\right), 73.7(\mathrm{C}), 113.8(\mathrm{CH}), 115.0(\mathrm{CH}), 118.7$ (CH), $128.6(\mathrm{CH}), 145.8(\mathrm{C}), 146.9$ (C) ppm. IR (KBr): 3420 (w), 3345 (w), 2896 (s), 2844 (s), 1615 (m), 1597 (m), 1489 (m), 1445 (m), $1416(\mathrm{w}), 1363(\mathrm{w}), 1346(\mathrm{w})$, 1313 (m), 1273 (w), 1103 (w), 994 (w), 781 (m), 694 (m), 452 (w) cm ${ }^{-1}$. GC-MS (EI, $70 \mathrm{eV}) ; \mathrm{m} / \mathrm{z}(\%): 41(6), 55(6), 67(6), 79$ (17), $81(5), 91(8), 93(13), 107(6), 135$ (23), 136 (12), 196 (100), 197 (12), $198(9), 345\left(\mathrm{M}^{+}, 5\right)$. 


\subsection{General procedure for preparation of anilines 25 and 26}

The corresponding aminodithiolane $(2.37 \mathrm{mmol})$ was dissolved in dioxane $(10 \mathrm{~mL})$ and large excess of Ra-Ni was added. The mixture was stirred and refluxed under an $\mathrm{Ar}$ atmosphere. If repeated $\mathrm{GC}$ analysis showed no significant progress, further portions of Ra-Ni were added until the starting material was completely consumed. Ra-Ni was filtered off, the filtrate was diluted with water and extracted several times with diethyl ether. Collected organic portions were washed with brine, dried over $\mathrm{Na}_{2} \mathrm{SO}_{4}$ and evaporated in vacuo. The desired crude product obtained as orange oil was subsequently converted to the hydrochloride.

4.9.1. 4-(1-Adamantylmethyl)anilinium chloride $(\mathbf{2 5} \cdot \mathbf{H C l})$ The crude product was dissolved in hexane/diethyl ether and $\mathbf{2 5} \cdot \mathbf{H C l}$ precipitated when dry $\mathrm{HCl}$ was introduced into solution. Yield: $579 \mathrm{mg}(88 \%)$ of a colourless microcrystalline powder. Mp $178-188^{\circ} \mathrm{C}, \mathrm{R}_{\mathrm{f}}\left(\right.$ free base) 0.28 (system b), anal. calcd for $\mathrm{C}_{17} \mathrm{H}_{24} \mathrm{ClN}$ : C, 73.49\%; H, 8.71\%; N, 5.04\%; found C, 73.22\%; H, 8.53\%; N, 4.81\%. ${ }^{1} \mathrm{H}$ NMR (DMSO- $\left.d_{6}\right): \delta 1.43$ (m, 6H, $\left.\mathrm{CH}_{2}(\mathrm{Ad})\right), 1.57$ (m, 6H, $\left.\mathrm{CH}_{2}(\mathrm{Ad})\right), 1.90(\mathrm{~m}, 3 \mathrm{H}$, CH(Ad)), 2.38 (s, 2H, AdCH $2 \mathrm{Ph}), 7.19$ (d, J=7.9 Hz, 2H, Ph), 7.31 (d, J=7.9 Hz, 2H, $\mathrm{Ph}$ ), 10.43 (bs, $3 \mathrm{H}, \mathrm{NH}_{3}{ }^{+}$) ppm. ${ }^{13} \mathrm{C}$ NMR (DMSO- $d_{6}$ ): $\delta 27.9(\mathrm{CH}), 32.9(\mathrm{C}), 36.4$ $\left(\mathrm{CH}_{2}\right), 41.6\left(\mathrm{CH}_{2}\right), 49.5\left(\mathrm{CH}_{2}\right), 122.5(\mathrm{CH}), 129.4(\mathrm{C}), 131.3(\mathrm{CH}), 137.7(\mathrm{C}) \mathrm{ppm}$. IR (KBr): 2902 (s), 2848 (s), 1613 (w), 1573 (w), 1509 (m), 1450 (w), 1314 (w), 1205 (w), $817(\mathrm{w}), 601(\mathrm{~m}), 526(\mathrm{w}), 485(\mathrm{~m}) \mathrm{cm}^{-1}$. GC-MS (EI, 70eV); m/z (\%): 41 (6), 55 (5), 67 (7), 77 (12), 79 (23), 81 (5), 91 (6), 93 (18), 106 (100), 107 (20), 135 (69), 136 (8), $241\left(\mathrm{M}^{+}, 35\right), 242(7)$. 


\subsubsection{3-[2-(1-Adamantyl)ethyl] anilinium chloride $(\mathbf{2 6} \cdot \mathbf{H C l})$ The crude product was} dissolved in hexane and $\mathbf{2 6} \cdot \mathbf{H C l}$ precipitated when dry $\mathrm{HCl}$ was introduced into solution. Yield: $491 \mathrm{mg}$ (71\%) of a colourless microcrystalline powder. Mp 142$150^{\circ} \mathrm{C}, \mathrm{R}_{\mathrm{f}}$ (free base) 0.25 (system b), anal. calcd for $\mathrm{C}_{18} \mathrm{H}_{26} \mathrm{ClN}$ : C, $74.07 \% ; \mathrm{H}$, $8.98 \%$;, $4.80 \%$; found C, 73.86\%; H, 9.17\%; N, 4.63\%. ${ }^{1} \mathrm{H}$ NMR (DMSO- $\left.d_{6}\right): \delta$ $1.30\left(\mathrm{~m}, 2 \mathrm{H}, \mathrm{AdCH}_{2}\right), 1.52\left(\mathrm{~m}, 6 \mathrm{H}, \mathrm{CH}_{2}(\mathrm{Ad})\right), 1.65\left(\mathrm{~m}, 3 \mathrm{H}, \mathrm{CH}_{2}(\mathrm{Ad}), 1.94(\mathrm{~s}, 3 \mathrm{H}\right.$, $\underline{\mathrm{CH}}(\mathrm{Ad})), 2.55(\mathrm{~m}, 2 \mathrm{H}, \mathrm{PhCH} 2), 7.22(\mathrm{~m}, 3 \mathrm{H}, \mathrm{Ph}), 7.36(\mathrm{t}, J=7.9 \mathrm{~Hz}, 1 \mathrm{H}, \mathrm{Ph})$, 10.37 (bs, 3H, NH $\left.{ }_{3}\right)$ ppm. ${ }^{13} \mathrm{C}$ NMR (DMSO- $\left.d_{6}\right): \delta 28.1(\mathrm{CH}), 28.2\left(\mathrm{CH}_{2}\right), 32.0(\mathrm{CH})$, $36.6\left(\mathrm{CH}_{2}\right), 41.7\left(\mathrm{CH}_{2}\right), 46.0\left(\mathrm{CH}_{2}\right), 120.3(\mathrm{CH}), 122.7(\mathrm{CH}), 127.8(\mathrm{CH}), 129.5$ (CH), $131.8(\mathrm{C}), 145.1$ (C) ppm. IR (KBr): 3429 (w), 2901 (s), 2845 (s), 2676 (w), $2605(\mathrm{~m}), 2362(\mathrm{w}), 1964(\mathrm{w}), 1598(\mathrm{~m}), 1576(\mathrm{w}), 1518(\mathrm{w}), 1490(\mathrm{~m}), 1451(\mathrm{~m})$, $1359($ w), $1314($ w), 1243 (w), $1101($ w), $1046(w), 790(w), 691(m), 524(w), 437$ (w) $\mathrm{cm}^{-1}$. GC-MS (EI, 70eV); m/z (\%): 41 (15), 53 (7), 55 (11), 67 (15), 77 (23), 78 (6), 79 (42), 80 (5), 91 (16), 93 (34), 94 (7), 105 (5), 106 (46), 107 (67), 119 (22), 120 (49), 121 (7), 135 (59), 136 (7), 149 (8), 255 ( $\left.\mathrm{M}^{+}, 100\right), 256$ (21).

\subsubsection{3-(1-Adamantylmethyl)anilinium chloride $(\mathbf{2 7} \cdot \mathbf{H C l})$ was prepared according to} the procedure used for previous amines. The nitrodithiane $16(338 \mathrm{mg}, 0.90 \mathrm{mmol})$ was dissolved in ethanol ( $40 \mathrm{~mL}$ ) under $\mathrm{H}_{2}$ atmosphere and large excess of Ra-Ni was added portionwise until starting material disappeared. The crude product was dissolved in hexane and $27 \cdot \mathbf{H C l}$ precipitated when dry $\mathrm{HCl}$ was introduced into solution. Yield: $188 \mathrm{mg}(75 \%)$ of a colourless microcrystalline powder. Mp 182$187^{\circ} \mathrm{C}, \mathrm{R}_{\mathrm{f}}$ (free base) 0.42 (system b), anal. calcd for $\mathrm{C}_{17} \mathrm{H}_{24} \mathrm{ClN}$ : C, $73.49 \%$; $\mathrm{H}$, $8.71 \%$; N, 5.04\%; found C, 73.62\%; H, 8.51\%; N, 5.24\%. ${ }^{1} \mathrm{H}$ NMR (DMSO- $\left.d_{6}\right): \delta$ 1.44 (m, 6H, $\left.\mathrm{CH}_{2}(\mathrm{Ad})\right), 1.50-1,66$ (m, 6H, $\left.\mathrm{CH}_{2}(\mathrm{Ad})\right), 1.91$ (m, 3H, $\left.\mathrm{CH}(\mathrm{Ad})\right), 2.38$ (s, 
2H, AdCH $\left.\mathbf{H}_{2} \mathrm{Ph}\right), 7.07-7.11(\mathrm{~m}, 2 \mathrm{H}, \mathrm{Ph}), 7.20(\mathrm{~d}, J=7.9 \mathrm{~Hz}, 1 \mathrm{H}, \mathrm{Ph}), 7.37$ (t, J=7.9 Hz,

1H, Ph), $10.14\left(\mathrm{bs}, 3 \mathrm{H}, \mathrm{NH}_{3}{ }^{+}\right.$) ppm. ${ }^{13} \mathrm{C}$ NMR (DMSO-d $): \delta 27.9(\mathrm{CH}), 32.9(\mathrm{C})$,

$36.4\left(\mathrm{CH}_{2}\right), 41.6\left(\mathrm{CH}_{2}\right), 49.8\left(\mathrm{CH}_{2}\right), 120.3(\mathrm{CH}), 124.4(\mathrm{CH}), 128.7(\mathrm{CH}), 129.6$

(CH), 131.8 (C), 139.4 (C) ppm. IR (KBr): 3421 (m), 2901 (s), 2846 (s), 2604 (m),

1602 (w), 1578 (m), 1518 (w), 1487 (m), 1452 (m), 1105 (w), 795 (m), $742(\mathrm{w}), 716$

(w), $694(\mathrm{~m}) \mathrm{cm}^{-1} . \mathrm{GC}-\mathrm{MS}(\mathrm{EI}, 70 \mathrm{eV}) ; \mathrm{m} / \mathrm{z}(\%): 41$ (6), 67 (8), 77 (8), 79 (19), 91 (5),

93 (16), 106 (11), 107 (13), 135 (100), $136(11), 241\left(\mathrm{M}^{+}, 35\right), 242(7)$.

\section{Acknowledgments}

This work was supported by Tomas Bata Foundation to RV, Ministry of Education, Youth and Sports of Czech Republic (Grant No. MSM7088352101 to RV and IK, MSM0021622413 and LC06030 to RM) and institutional research plan AV0Z40310501 to RC.

\section{Supplementary material}

Supplementary data associated with this article can be found in online version at ...

These data include NMR data obtained from titration of guest 15 by $\beta$-CD and data obtained from isothermal titration calorimetry for guest 7 .

\section{References}

1. Davies, W. L.; Grunert, R. R.; Haff, R. F.; McGahen, J. W.; Neumayer, E. M.; Paulshock, M.; Wats, J. C.; Wood, T. R.; Hermann, E. C.; Hoffmann, C. E. Antiviral activity of 1-adamantanamine (amantadine). Science 1964, 144, 862-863.

2. (a) Stamatiou, G.; Foscolos, G. B.; Fytas, G.; Kolocouris, A.; Kolocouris, N.; Pannecouque, C.; Witvrouw, M.; Padalko, E.; Neyts, J.; de Clercq, E. Heterocyclic rimantadine analogues with antiviral activity. Bioorg. Med. Chem. 2003, 11, 5485-5492; (b) Wagner, C. E.; Mohler, M. L.; Kang, G. S.; Miller, D. D.; Geisert, E. E.; Chang, Y.-A.; Fleischer, E. B.; Shea, K. J. Synthesis of 1-Boraadamantaneamine Derivatives with Selective Astrocyte vs. C6 Glioma Antiproliferative Activity. A Novel Class of Anti-Hepatitis C Agents with Potential to Bind CD81. J. Med. Chem. 2003, 46, 2823-2833; (c) Tataridis, D.; Fytas, G.; Kolocouris, A.; Fytas, C.; Kolocouris, N.; Foscolos, G. B.; Padalko, E.; Neyts, J.; De Clerq, E. Influence of an additional 2-amino substituent of the 1-aminoethyl pharmacophore group on the potency of rimantadine against influenza virus A. Bioorg. Med. Chem. Lett. 2007, 17, 692-696; (d) Zoidis, G.; Fytas, C.; Papanastasiou, I.; Foscolos, G. B.; Fytas, G.; Padalko, E.; de Clercq, E.; Naesens, L.; Neyts, J.; Kolocouris, N.

Heterocyclic rimantadine analogues with antiviral activity. Bioorg. Med. Chem. 2006, 14, 3341-3348; (e) Makarova, N. V.; Boreko, E. I.; Moiseev, I. K.; Pavlova, N. I.; Zemtsova, M. N.; Nikolaeva, S. N.; Vladyko, G. V. 
Antiviral activity of adamantyl-containing $\beta$-aminoketones, enaminoketones, and related compounds. Pharm. Chem. J. 2001, 35, 480-484; (f) Motornaya, A. E.; Alimbarova, L. M.; Shokova, É. A.; Kovalev, V. V. Synthesis and antiherpetic activity of N-(3-amino-1-adamantyl)calix[4]arenes. Pharm. Chem. J. 2006, 40, 68-72.

| $3 . \quad$ (a) Long, J.; Manchandia, T.; Ban, K.; Gao, S.; Miller, C.; Chandra, J. Adaphostin cytotoxicity in glioblastoma cells is ROS-dependent and is accompanied by upregulation of heme oxygenase-1. Cancer Chemother. Pharmacol. 2007, 59, 527-535; (b) Roubalová, E.; Kvardová, V.; Hrstka, R.; Bořilová, Š.; Michalová, E.; Dubská, L.; Müiller, P.; Sova, P.; Vojtěšek, B. The effect of cellular environment and p53 status on the mode of action of the platinum derivative LA-12. Invest. New Drugs 2010, 28, 445-453.

4. (a) El-Emam, A. A.; Al-Deeb, O. A.; Al-Omar, M.; Lehmann, J. Synthesis, antimicrobial, and anti-HIV-1 activity of certain 5-(1-adamantyl)-2-substituted thio-1,3,4-oxadiazoles and 5-(1-adamantyl)-3-substituted aminomethyl-1,3,4oxadiazoline-2-thiones. Bioorg. Med. Chem. 2004, 12, 5107-5113; (b) Kadi, A. A.; El-Brollosy, N. R.; Al-Deeb, O. A.; Habib, E. E.; Ibrahim, T. M.; El.Emam, A. A. Synthesis, antimicrobial, and anti-inflammatory activities of novel 2-(1-adamantyl)-5-substituted-1,3,4-oxadiazoles and 2-(1adamantylamino)-5-substituted-1,3,4-thiadiazoles. Eur. J. Med. Chem. 2007, 42, 235-242.

5. (a) Koo, K. D.; Kim, M. J.; Kim, S.; Kim, K.-H.; Hong, S. Y.; Hur, G.-C.; Yim, H. J.; Kim, G. T.; Han, H. O.; Kwon, O. H.; Kwon, T. S.; Koh, J. S.; Lee, Ch.-S. Synthesis, SAR, and X-ray structure of novel potent DPPIV inhibitors: Oxadiazolyl ketones. Bioorg. Med. Chem. Lett. 2007, 17, 41674172; (b) Villhauer, E. B.; Brinkman, J. A.; Naderi, G. B.; Burkey, B. F.; Dunning, B. E.; Prasad, K.; Mangold, B. L.; Russell, M. E.; Hughes, T. E. 1[[(3-Hydroxy-1-adamantyl)amino]acetyl]-2-cyano-( $S)$-pyrrolidine: A Potent, Selective, and Orally Bioavailable Dipeptidyl Peptidase IV Inhibitor with Antihyperglycemic Properties. J. Med. Chem. 2003, 46, 2774-2789; (c) Ahrén, B.; Landin-Olsson, M.; Jansson, P.-A.; Svensson, M.; Holmes, D.; Schweizer, A. Inhibition of dipeptidyl peptidase-4 reduces glycemia, sustains insulin levels, and reduces glucagon levels in type 2 diabetes. J. Clin. Endocr. Metab. 2004, 89, 2078-2084.

6. (a) Farhana, L.; Dawson, M. I.; Leid, M.; Wang, L.; Moore, D. D.; Liu, G.; Xia, Z.; Fontana, J. A. Adamantyl-substituted retinoid-related molecules bind small heterodimer partner and modulate the Sin3A repressor. Cancer Res. 2007, 67, 318-327; (b) Farhana, L.; Dawson, M. I.; Fontana, J. A. Apoptosis Induction by a Novel Retinoid-Related Molecule Requires Nuclear Factor- $\kappa \mathrm{B}$ Activation. Cancer Res. 2005, 65, 4909-4917; (c) Parrella, E.; Gianni, M.; Fratelli, M.; Barzago, M. M.; Raska Jr., I.; Diomede, L.; Kurosaki, M.; Pisano, C.; Carminati, P.; Merlini, L.; Dallavalle, S.; Tavecchio, M.; Rochette-Egly, C.; Terao, M.; Garattini, E. Antitumor activity of the retinoid-related molecules (E)-3-(4'-hydroxy-3'-adamantylbiphenyl-4-yl)acrylic acid (ST1926) and 6-[3-(1-adamantyl)-4-hydroxyphenyl]-2-naphthalene carboxylic acid (CD437) in F9 teratocarcinoma: role of retinoic acid receptor $\gamma$ and retinoidindependent pathways. Mol. Pharmacol. 2006, 70, 909-924; (d) Wanner, R.; Henseleit-Walter, U.; Wittig, B.; Kolde, G. Proliferation-dependent induction of apoptosis by the retinoid CD437 in p53-mutated keratinocytes. J. Mol. Med. 2002, 80, 61-67.

Deleted:; (e) Dawson, M. I.; Xia, Z.;
Jiang, T.; Ye, M.; Fontana, J. A.;
Farhana, L.; Patel, B.; Xue, L. P.;
Bhuiyan, M.; Pellicciari, R.; Macchiarulo,
A.; Nuti, R.; Zhang, X.-K.; Han, Y.-H.;
Tautz, L.; Hoobs, P. D.; Jong, L.; Waleh,
N.; Chao, W.; Feng, G.-S.; Pang, Y.; Su,
Y. Adamantyl-Substituted Retinoid-
Derived Molecules That Interact with the
Orphan Nuclear Receptor Small
Heterodimer Partner: Effects of
Replacing the 1-Adamantyl or Hydroxyl
Group on Inhibition of Cancer Cell
Growth, Induction of Cancer Cell
Apoptosis, and Inhibition of Src
Homology 2 Domain-Containing Protein
Tyrosine Phosphatase-2 Activity. J. Med.
Chem. 2008, 51, 5650-5662; (f)
Keedwell, R. G.; Zhao, Y.; Hammond, L.
A.; Qin, S.; Tsang, K.-Y.; Reitmair, A.;
Molina, Y.; Okawa, Y.; Atangan, L. I.;
Shurland, D.-L.; Wen, K.; Wallace, D. M.
A.; Bird, R.; Chandraratna, R. A. S.;
Brown, G. A Retinoid-Related Molecule
that Does Not Bind to Classical Retinoid
Receptors Potently Induces Apoptosis in
Human Prostate Cancer Cells through
Rapid Caspase Activation. Cancer Res.
2004, 64, 3302-3312.


7. (a) Jensen, L. S.; Bølcho, U.; Egebjerg, J.; Strømgaard, K. Design, synthesis, and pharmacological characterization of polyamine toxin derivatives: potent ligands for the pore-forming region of AMPA receptors. ChemMedChem 2006, 1, 419-428; (b) Schlesinger, F.; Tammena, D.; Krampfl, K.; Bufler, J. Two mechanisms of action of the adamantane derivative IEM-1460 at human AMPA-type glutamate receptors. Brit. J. Pharmacol. 2005, 145, 656-663; (c) Wang, Y.; Eu, J.; Washburn, M.; Gong, T.; Chen, H.-S. V.; James, W. L.; Lipton, S. A.; Stamler, J. S.; Went, G. T.; Porter, S. The pharmacology of aminoadamantane nitrates. Curr. Alzheimer Res. 2006, 3, 201-204.

8. (a) Hwang, S. H.; Morisseau, Ch.; Do, Z.; Hammock, B. D. Solid-phase combinatorial approach for the optimization of soluble epoxide hydrolase inhibitors. Bioorg. Med. Chem. Lett. 2006, 16, 5773-5777; (b) Anandan, S.K.; Do, Z. N.; Webb, H. K.; Patel, D. V.; Gless, R. D. Non-urea functionality as the primary pharmacophore in soluble epoxide hydrolase inhibitors. Bioorg. Med. Chem. Lett. 2009, 19, 1066-1070; (c) Kim, I.-H.; Morisseau, Ch.; Watanabe. T.; Hammock, B. D. Design, Synthesis, and Biological Activity of 1,3-Disubstituted Ureas as Potent Inhibitors of the Soluble Epoxide Hydrolase of Increased Water Solubility. J. Med. Chem. 2004, 47, 2110-2122; (d) Hwang, S. H.; Tsai, H.-J.; Liu, J.-Y.; Morisseau, Ch.; Hammock, B. D. Orally Bioavailable Potent Soluble Epoxide Hydrolase Inhibitors. J. Med. Chem. 2007, 50, 3825-3840.

9. (a) Lee, R. E.; Protopopova, M.; Crooks, E.; Slayden, R. A.; Terrot, M.; Barry, C. E., III Combinatorial Lead Optimization of [1,2]-Diamines Based on Ethambutol as Potential Antituberculosis Preclinical Candidates. J. Comb. Chem. 2003, 5, 172-187; (b) Nayyar, A.; Monga, V.; Malde, A.; Coutinho, E.; Jain, R. Synthesis, anti-tuberculosis activity, and 3D-QSAR study of 4(adamantan-1-yl)-2-substituted quinolines. Bioorg. Med. Chem. 2007, 15, 626-640.

10. (a) Lu, D.; Meng, Z.; Thakur, G. A.; Fan, P.; Steed, J.; Tartal, C. L.; Hurst, D. P.; Reggio, P. H.; Deschamps, J. R.; Parrish, D. A.; George, C.; Järbe, T. U. C.; Lamb, R. J.; Makriyannis, A. Adamantyl Cannabinoids: A Novel Class of Cannabinergic Ligands. J. Med. Chem. 2005, 48, 4576-4585; (b) Stern, E.; Muccioli, G. G.; Bosier, B.; Hamtiaux, L.; Millet, R.; Poupaert, J. H.; Hénichart, J.-P.; Depreux, P.; Goossens, J.-F.; Lambert, D. M. Pharmacomodulations around the 4-Oxo-1,4-dihydroquinoline-3carboxamides, a Class of Potent CB2-Selective Cannabinoid Receptor Ligands: Consequences in Receptor Affinity and Functionality. J. Med. Chem. 2007, 50, 5471-5484.

11. (a) Cromwell, W. C.; Byström, K.; Efting, M. R. Cyclodextrinadamantanecarboxylate inclusion complexes: studies of the variation in cavity size. J. Phys. Chem. 1985, 89, 326-332; (b) van Bommel, K. J. C.; Metselaar, G. A.; Verboom, W.; Reinhoudt, D. N. Water-Soluble Adamantane-

Terminated Dendrimers Possessing a Rhenium Core. J. Org. Chem. 2001, 66, 5405-5412.

12. (a) Uekama, K.; Hirayama, F.; Irie, T. Cyclodextrin Drug Carrier Systems. Chem. Rev. 1998, 98, 2045-2076 and references therein; (b) Brewster, M. E.; Loftsson, T. Cyclodextrins as pharmaceutical solubilizers. Adv. Drug Delivery Rev. 2007, 59, 645-666; (c) Okáčová, L.; Vetchý, D.; Franc, A.; Rabišková, M. Increasing bioavailability of poorly water-soluble drugs by technological methods facilitating drug dissolution. Chem. Listy 2011, 105, 34-40. 
13. (a) Otyepka, M.; Kryštof, V.; Havlíček, L.; Siglerová, V.; Strnad, M.; Koča, J. Docking-Based Development of Purine-like Inhibitors of Cyclin-Dependent Kinase-2. J. Med. Chem. 2000, 43, 2506-2513; (b) Barbarić, M.; Uršić, S.; Pilepić, V.; Zorc, B.; Hergold-Brundić, A.; Nagl, A.; Grdiša, M.; Pavelić, K.; Snoeck, R.; Andrei, G.; Balzarini, J.; de Clercq, E.; Mintas, M. Synthesis, Xray crystal structure study, and cytostatic and antiviral evaluation of the novel cycloalkyl-N-aryl-hydroxamic acids. J. Med. Chem. 2005, 48, 884-887.

14. (a) Bistri, O.; Mazeau, K.; Auzély-Velty, R.; Sollogoub, M. Chemical clockwise tridifferentiation of $\alpha$ - and $\beta$-cyclodextrins: Bascule-bridge or deoxy-sugars strategies. Chem. Eur. J. 2007, 13, 8847-8857; (b) Rüdiger, V.; Eliseev, A.; Simova, S.; Schneider, H.-J.; Blandamer, M. J.; Cullis, P. M.; Meyer, A. J. Conformational, calorimetric and NMR spectroscopic studies on inclusion complexes of cyclodextrins with substituted phenyl and adamantane derivatives. J. Chem. Soc. Perkin Trans. 2 1996, 2119-2123; (c) Carrazana, J.; Jover, A.; Meijide, F.; Soto, V. H.; Tato, J. V. Complexation of Adamantyl Compounds by $\beta$-Cyclodextrin and Monoaminoderivatives. J. Phys. Chem. B 2005, 109, 9719-9726; (d) Hamilton, J. A.; Sabesan, M. N. Structure of a complex of cycloheptaamylose with 1-adamantanecarboxylic acid. Acta Crystallogr., Sect. B: Struct. Sci. 1982, 38, 3063-3069; (e) Weickenmeier, M.; Wenz, G. Cyclodextrin side chain polyesters. Synthesis and inclusion of adamantane derivatives. Macromol. Rapid Commun. 1996, 17, 731-736.

15. (a) Leclerq, L.; Schmitzer, A. R. Supramolecular encapsulation of 1,3-bis(1adamantyl)imidazolium chloride by $\beta$-cyclodextrins: towards inhibition of C(2)-H/D exchange. J. Phys. Org. Chem. 2009, 22, 91-95; (b) Leggio, C.; Anselmi, M.; di Nola, A.; Galantini, L.; Jover, A.; Meijide, F.; Pavel, N. V.; Tellini, V. H. S.; Tato, J. V. Study on the Structure of Host-Guest Supramolecular Polymers. Macromolecules 2007, 40, 5899-5906; (c) Ohga, K.; Takashima, Y.; Takahashi, H.; Kawaguchi, Y.; Yamaguchi, H.; Harada, A. Preparation of supramolecular polymers from a cyclodextrin dimer and ditopic guest molecules: control of structure by linker flexibility. Macromolecules, 2005, 38, 5897-5904; (d) Tellini, V. H. S.; Jover, A.; Galantini, L.; Meijide, F.; Tato, J. V. Crystal structure of the supramolecular linear polymer formed by the self-assembly of mono-6-deoxy-6-adamantylamide- $\beta$-cyclodextrin. Acta Crystallogr., Sect. B: Struct. Sci. 2004, 60, 204-210; (e) Taura, D.; Taniguchi, Y.; Hashidzume, A.; Harada, A. Macromolecular Recognition of Cyclodextrin: Inversion of Selectivity of $\beta$-Cyclodextrin toward Adamantyl Groups Induced by Macromolecular Chains. Macromol. Rapid Commun. 2009, 30, 1741-1744; (f) Liu, H.; Zhang, Y.; Hu, J.; Li, C.; Liu, S. MultiResponsive Supramolecular Double Hydrophilic Diblock Copolymer Driven by Host-Guest Inclusion Complexation between $\beta$-Cyclodextrin and Adamantyl Moieties. Macromol. Chem. Phys. 2009, 210, 2125-2137.

16. Vícha, R.; Potáček, M. Influence of catalytic system composition on formation of adamantane containing ketones. Tetrahedron 2005, 61, 83-85.

17. Vícha, R.; Kuřitka, I.; Rouchal, M.; Ježková, V.; Zierhut, A. Directing effects in nitration of 1-adamantyl bearing aromatic ketones. ARKIVOC 2009, XII, 60-80.

18. Rouchal, M.; Nečas, M.; Vícha, R. (1-Adamantyl)(4-aminophenyl)methanol. Acta Crystallogr., Sect. E: Struct. Rep. Online 2009, 65, o1018.

19. (a) Dodziuk H. In Cyclodextrins and their complexes; Dodziuk, H., Ed.; Wiley-VCH: Weinheim, 2006; Chapter 1 and references therein; (b) Seidel, R.

Deleted: (b) Szejtli, J. Introduction and General Overview of Cyclodextrin Chemistry. Chem. Rev. 1998, 98, 17431753 and references therein;

Deleted: c 
W.; Koleva, B. B. B-Cyclodextrin 10.41-hydrate. Acta Crystallogr., Sect. E: Struct. Rep. Online 2009, 65, o3162-o3163; (c) Schneider, H.-J.; Hacket, F.; Rüdiger, V.; Ikeda, H. NMR Studies of Cyclodextrins and Cyclodextrin Complexes. Chem. Rev. 1998, 98, 1755-1785.

20. Stewart, J. J. P. Optimization of parameters for semiempirical methods. II. Applications. J. Comput. Chem. 1989, 10, $221-264$.

| 21. Britto, M.; Nascimento, C. S., Jr.; Dos Santos, H. F. Structural analysis of cyclodextrins: a comparative study of classical and quantum mechanical methods. Quim. Nova 2004 27, 882-888.

| 22. Zheng, Y. J.; Merz, K.M. Study of hydrogen bonding interactions relevant to biomolecular structure and function. J. Comput. Chem. 1992, $13,1151-1169$.

| 23. Yang, E. C.; Zhao, X. J.; Hua, F.; Hao, J. K. Semi-empirical PM3 study upon the complexation of $\beta$-cyclodextrin with 4,4'-benzidine and o-tolidine. J. Mol. Struct.: THEOCHEM 2004, 712, 75-79.

| 24. Rouchal, M.; Nečas, M.; Vícha, R. 1-(2-Phenylethyl)adamantane. Acta Crystallogr., Sect. E: Struct. Rep. Online 2010, 66, o1736.

| 25. Jeener, J.; Meier, B. H.; Bachmann, P.; Ernst, R. R. Investigation of exchange processes by two-dimensional NMR spectroscopy. J. Chem. Phys. 1979, 71 , 4546-4553.

| 26. Martin, G. E.; Crouch, R. C. Inverse-detected two-dimensional NMR methods: applications in natural products chemistry. J. Nat. Prod. 1991, $\overline{4}$, 1-70.

| 27. Kay, L. E.; Keifer, P.; Saarinen, T. Pure absorption gradient enhanced heteronuclear single quantum correlation spectroscopy with improved sensitivity. J. Am. Chem. Soc. 1992, 114, 10663-10665.

28. SPARTAN'08, version 1.2.0. Wavefunction, Inc., Irvine, CA, USA, 2008. Jursic, B. S.; Zdravkovski, Z.; French, A. D. Molecular modeling methodology of $\beta$-cyclodextrin inclusion complexes. J. Mol. Struct.: THEOCHEM 1996, 366, 113-117.
Deleted: d

Deleted: 20. . Saenger, W.; Jakob, J.; Gessler, K.; Steiner, T.; Hoffmann, D. Sanbe, H.; Koizumi, K.; Smith, S. M.; Tahaka, T. Structures of the Common Cyclodextrins and Their Larger AnalogsBeyond the Doughnut. Chem. Rev. 1998, 98, 1787-1802.II

21. . Fielding, L. Determination of Association Constants $\left(\mathrm{K}_{\mathrm{a}}\right)$ from Solution NMR Data. Tetrahedron 2000, 56, 61516170.II

Deleted: 2

Deleted: 23. Sheehan, R.; Cragg, P. J. Supramolecular Chemistry In Silico. Supramol. Chem. 2008. 20, 443-451.II

Deleted: 4

Deleted: 25

Deleted:

Deleted: 6

Deleted: 27

Deleted: 28

Deleted: 29

Deleted: 30 
Table 1. Results of MS analyses - ionic species observed for amine with and without the presence of $\beta-C D$

\begin{tabular}{|c|c|c|c|c|c|c|}
\hline \multirow[b]{2}{*}{ Amine } & \multicolumn{6}{|c|}{ Exact mass } \\
\hline & calc. & found & calc. & found & calc. & found \\
\hline 6 & 256.2 & 256.1 & 533.4 & 533.3 & 1390.6 & 1390.6 \\
\hline 7 & 256.2 & 256.1 & 533.4 & 533.3 & 1390.6 & 1390.6 \\
\hline 8 & 270.2 & 270.1 & 561.4 & 561.3 & 1404.6 & 1404.6 \\
\hline 12 & 272.2 & 272.1 & 565.4 & - & 1406.6 & 1406.6 \\
\hline 13 & 258.2 & 258.1 & 537.4 & 537.2 & 1392.6 & 1392.6 \\
\hline 14 & 272.2 & 272.1 & 565.4 & 565.4 & 1406.6 & 1406.7 \\
\hline 15 & 258.2 & 258.1 & 537.4 & 537.2 & 1392.6 & 1392.6 \\
\hline 21 & 346.2 & 346.1 & 714.4 & - & 1480.6 & 1480.7 \\
\hline 22 & 360.2 & 360.2 & 742.4 & - & 1494.6 & 1494.7 \\
\hline 24 & 346.2 & 346.3 & 714.4 & - & 1480.6 & 1480.8 \\
\hline 25 & 242.2 & 242.8 & 505.4 & - & 1376.6 & 1376.7 \\
\hline 26 & 256.2 & 256.3 & 533.4 & - & 1390.6 & 1390.7 \\
\hline 27 & 242.2 & 242.1 & 505.4 & - & 1376.6 & 1376.6 \\
\hline
\end{tabular}


Table 2. Thermodynamic parameters for inclusion complex formation of guest molecules and $\beta-\mathrm{CD}$, derived from calorimetric titration experiments in $\mathrm{DMSO} /$ water $(3 / 1, v / v)$ mixture at $30^{\circ} \mathrm{C}$.

\begin{tabular}{ccccc}
\hline guest & $\mathrm{K}\left[\mathrm{M}^{-1}\right]$ & $-\Delta \mathrm{H}\left[\mathrm{kJ} \cdot \mathrm{mol}^{-1}\right]$ & $-\Delta \mathrm{S}\left[\mathrm{J} \cdot \mathrm{K}^{-1} \cdot \mathrm{mol}^{-1}\right]$ & $\mathrm{n}$ \\
\hline $\mathbf{6}$ & $186 \pm 23$ & $35 \pm 14$ & 71 & $1.1 \pm 0.4$ \\
$\mathbf{7}$ & $226 \pm 25$ & $46 \pm 15$ & 105 & $1.0 \pm 0.3$ \\
$\mathbf{8}$ & $313 \pm 55$ & $38 \pm 17$ & 75 & $1.0 \pm 0.4$ \\
$\mathbf{2 6}$ & $694 \pm 28$ & $44 \pm 3$ & 88 & $0.93 \pm 0.05$
\end{tabular}


1

2

3

4

5

6

7

8

9

10

11

12

13

14

15

16

17

18

19

20

21

Table 3. Selected geometric parameters and free energies for complexes of amines $\mathbf{1 4}$, 26 and parent hydrocarbon PH with $\beta$-CD.

\begin{tabular}{cccccc}
\hline Structure & $d[\mathrm{~nm}]^{\mathrm{a}}$ & $l[\mathrm{~nm}]$ & $\alpha\left[^{\circ}\right]$ & $\beta\left[^{\circ}\right]$ & $\begin{array}{c}\text { Stabilisation } \\
\text { energy }[\mathrm{kJ} / \mathrm{mol}]\end{array}$ \\
\hline 14-SI & +0.0295 & 0.0227 & 142.48 & 50.31 & -613.62 \\
14-PI & -0.1534 & 0.1534 & 163.49 & 90.00 & -604.14 \\
26-SI & +0.0656 & 0.0622 & 16.10 & 71.47 & -655.29 \\
26-SE & +0.1173 & 0.1158 & 36.74 & 80.83 & -633.66 \\
PH-SI & +0.0930 & 0.0856 & 139.54 & 66.99 & -668.04 \\
PH-SE & +0.2223 & 0.2199 & 30.25 & 81.57 & -650.19 \\
\hline
\end{tabular}

I For the definition of $C g 1, C g 2, O g$, and $P$, see the experimental part $d$ is $C g 1-O g$ distance, $l$ is $C g 1-P$ distance, $\alpha$ is $C g 1-O g-C g 2$ angle, $\beta$ is $C g 1-O g-P$ angle. ${ }^{a}$ The positive or negative sign implies location of adamantane cage in cavity close to the secondary or primary rim, respectively.

Deleted: $C g 1$ is centre of mass of four bridgehead carbons in adamantane; $O g$ and $P$ are centres of mass and the best least squares plane of seven glycosidic oxygen atoms in $\beta$-cyclodextrin,

respectively; $\mathrm{Cg} 2$ is centre of mass of six carbons in benzene ring

Formatted: Font: Italic 
Scheme 1. Reaction pathway leading to 1-adamantyl (nitrophenyl) ketones.

Scheme 2. Synthesis of aminoketones and aminoalcohols.

Scheme 3. Synthesis of anilines with non-polar linker.

\section{Figure 1. Structural formulas for selected promising anticancer drugs.}

Figure 2. Typical ESI-MS data for studied amines (a), mixtures of amine and $\beta-\mathrm{CD}$ (b), and $\mathrm{MS}^{2}$ spectrum of amine $\beta-\mathrm{CD}$ complexes (c).

Deleted: 1

Figure 3. Schematic representations of $\beta$-CD and the prepared guest molecules with Deleted: 2 dimensions shown in $\mathrm{nm}$.

| Figure 4. Schematic representations of possible geometries of host-guest systems

Deleted: 3 under consideration. $\mathrm{S}=$ secondary, $\mathrm{P}=$ primary, $\mathrm{I}=$ internal, $\mathrm{E}=$ external. (Previously published ${ }^{14 \mathrm{c}}$ geometric parameters were considered.)

Figure 5. A portion of the NOESY spectrum of a 1:1 mixture of guest $\mathbf{1 4}$ with $\beta$-CD Deleted: 4 (left); A portion of the gs-HMQC-NOESY spectrum of a 1:1 mixture of guest 7 with $\beta-C D$ (middle and right). Detailed comment may be found in the text. Signals of host and guest nuclei are labelled as $\beta-\mathrm{CD}$ and $\mathrm{G}$, respectively.

Figure 6. Minimised structures of complexes of $\beta-C D$ with amine 26 and 14 , Deleted: 5 respectively. 


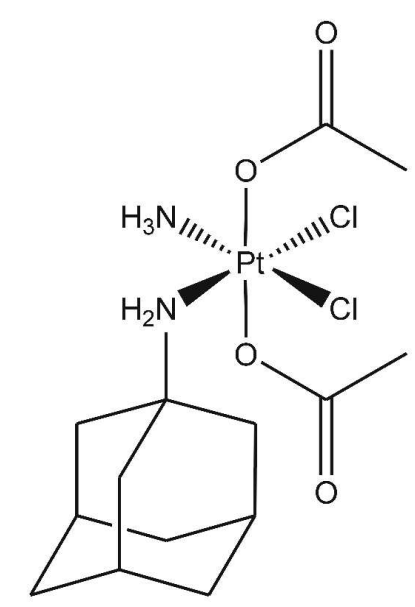

LA-12

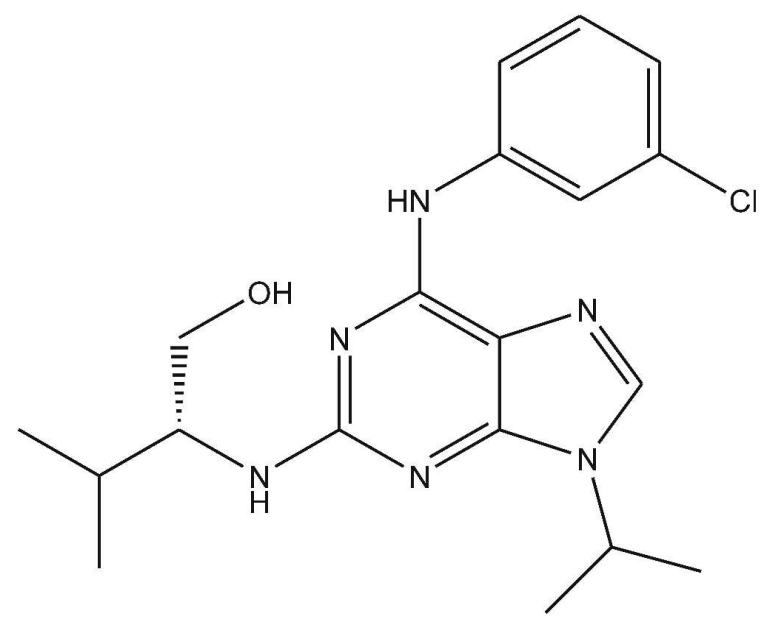

Purvalanol A

25

26

27

28

29

30

31

32

33

34

35

36

37

38

39

40

41

42

43

44

45

46

47

48

49

50

51

52

53

54

55

56

57

58

59

60

URL: http:/mc.manuscriptcentral.com/tandf/gsch Email: suprachem@mail.cm.utexas.edu 

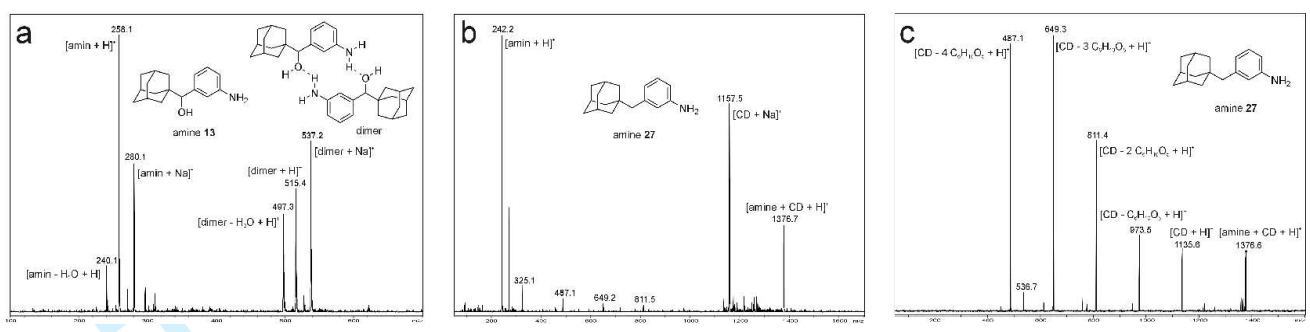
Typical ESI-MS data for studied amines (a), mixtures of amine and $\beta-C D(b)$, and MS2 spectrum of amine $\bullet \beta-C D$ complexes (c). 
Typical ESI-MS data for studied amines (a), mixtures of amine and $\beta-C D(b)$, and MS2 spectrum of amine $\beta$-CD complexes (c). 
Typical ESI-MS data for studied amines (a), mixtures of amine and $\beta-C D(b)$, and MS2 spectrum of amine $\bullet \beta-C D$ complexes (c). 

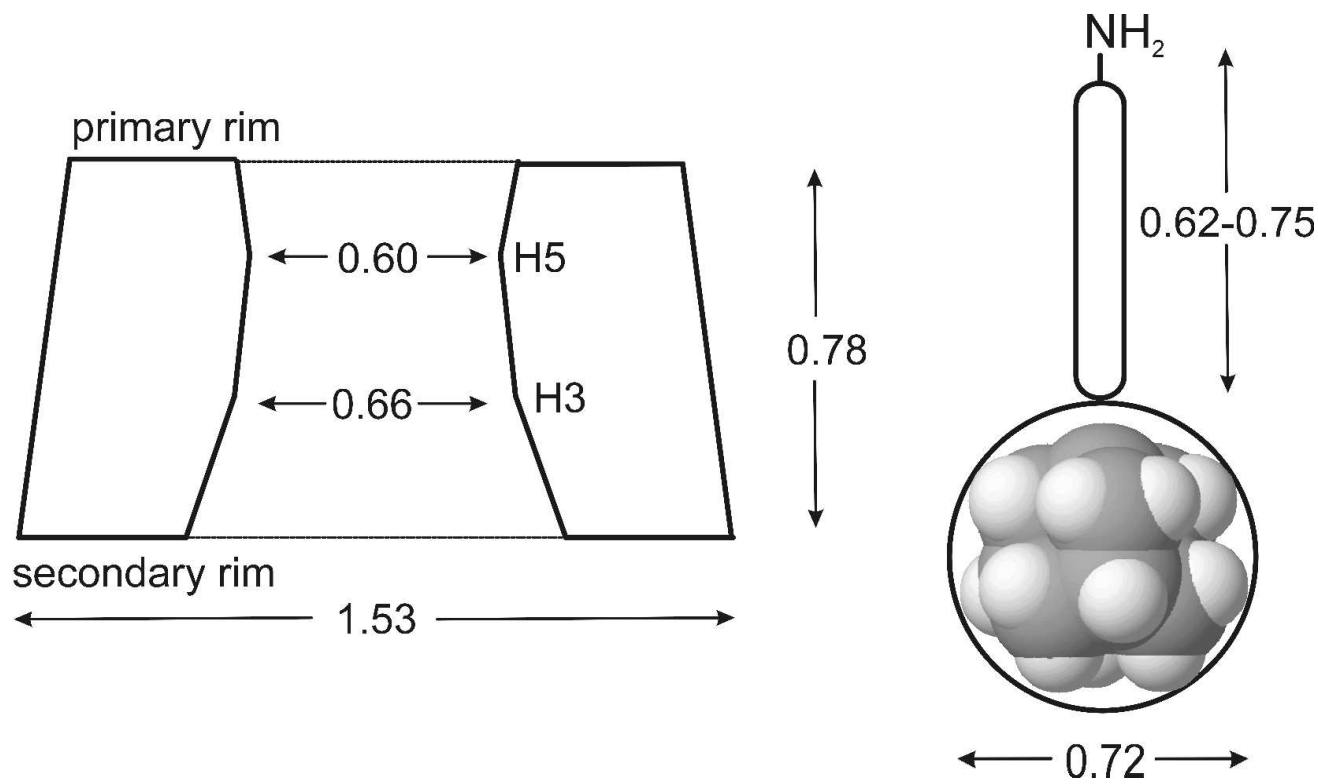

Schematic representations of $\beta-C D$ and the prepared guest molecules with dimensions shown in $\mathrm{nm}$. 
1

2

3

4

5

6

7

8

9

10

11

12

13

14

15

16

17

18

19

20

21

22

23

24

25

26

27

28

29

30

31

32

33

34

35

36

37

38

39

40

41

42

43

44

45

46

47

48

49

50

51

52

53

54

55

56

57

58

59

60

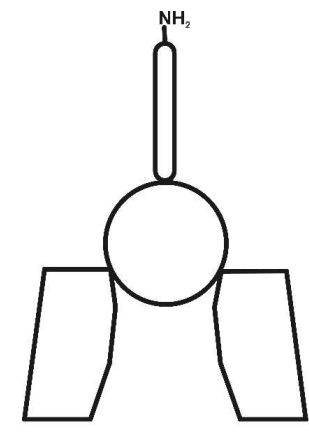

PE

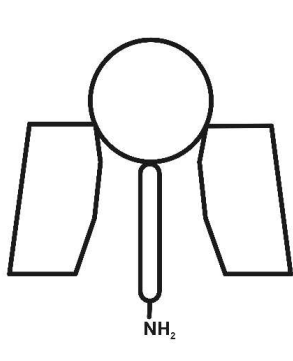

PI

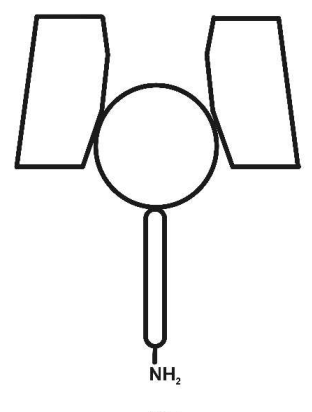

SE

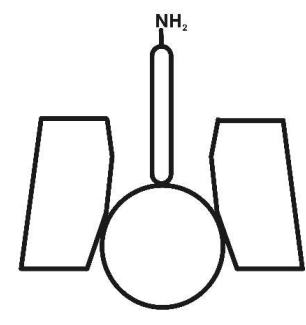

SI

Schematic representations of possible geometries of host-guest systems under consideration. $\mathrm{S}=$ secondary, $\mathrm{P}=$ primary, $\mathrm{I}=$ internal, $\mathrm{E}=$ external. (Previously published ${ }^{14 \mathrm{c}}$ geometric parameters were considered.) 
A portion of the NOESY spectrum of a $1: 1$ mixture of guest 14 with $\beta$-CD (left); A portion of the gsHMQC-NOESY spectrum of a 1:1 mixture of guest 7 with $\beta-C D$ (middle and right). Detailed comment may be found in the text. Signals of host and guest nuclei are labelled as $\beta-C D$ and $G$, respectively. 
1

2

3

4

5

6

7

8

9

10

11

12

13

14

15

16

17

18

19

20

21

22

23

24

25

26

27

28

29

30

31

32

33

34

35

36

37

38

39

40

41

42

43

44

45

46

47

48

49

50

51

52

53

54

55

56

57

58

59

60

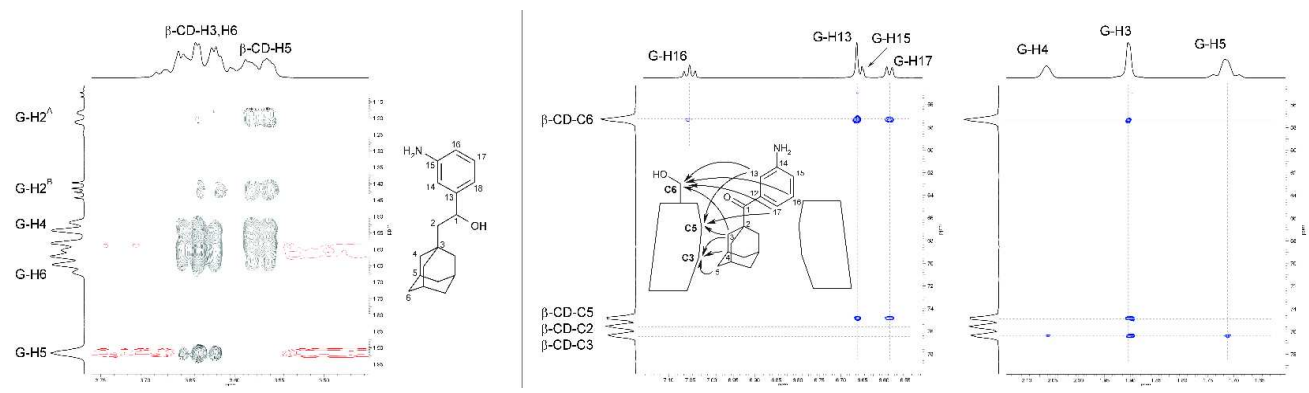

A portion of the NOESY spectrum of a $1: 1$ mixture of guest 14 with $\beta$-CD (left); A portion of the gsHMQC-NOESY spectrum of a $1: 1$ mixture of guest 7 with $\beta-C D$ (middle and right). Detailed comment may be found in the text. Signals of host and guest nuclei are labelled as $\beta-C D$ and $G$, respectively.

URL: http:/mc.manuscriptcentral.com/tandf/gsch Email: suprachem@mail.cm.utexas.edu 


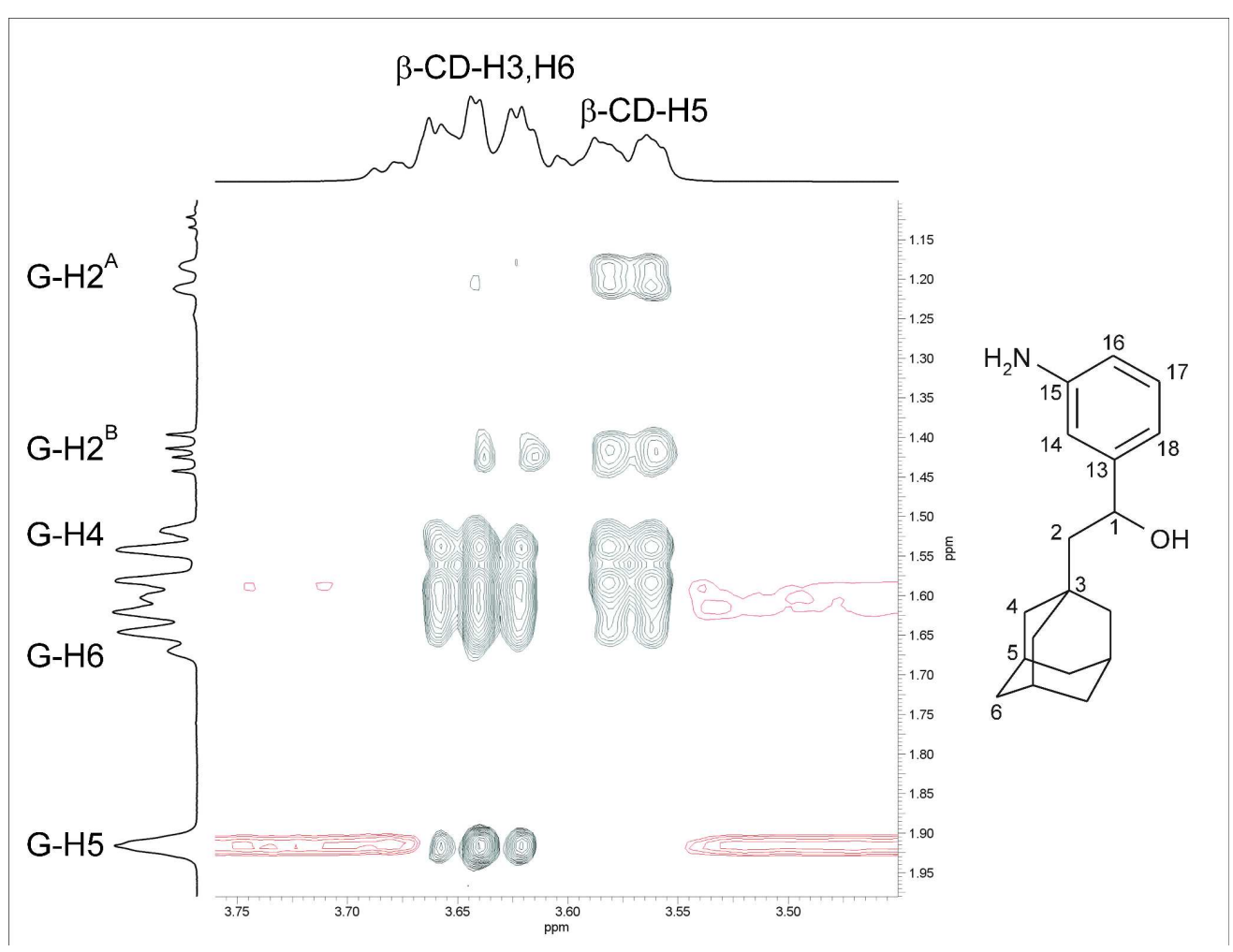

A portion of the NOESY spectrum of a $1: 1$ mixture of guest 14 with $\beta-C D$ (left); A portion of the gsHMQC-NOESY spectrum of a 1:1 mixture of guest 7 with $\beta-C D$ (middle and right). Detailed comment may be found in the text. Signals of host and guest nuclei are labelled as $\beta-C D$ and G, respectively. 


\section{Unable to Convert Image}

The dimensions of this image (in pixels) are too large to be converted. For this image to convert, the total number of pixels (height $x$ width) must be less than 40,000,000 (40 megapixels).

\footnotetext{
A portion of the NOESY spectrum of a $1: 1$ mixture of guest 14 with $\beta-C D$ (left); A portion of the gsHMQC-NOESY spectrum of a 1:1 mixture of guest 7 with $\beta-C D$ (middle and right). Detailed comment may be found in the text. Signals of host and guest nuclei are labelled as $\beta-C D$ and $G$, respectively.
} 
A portion of the NOESY spectrum of a $1: 1$ mixture of guest 14 with $\beta$-CD (left); A portion of the gsHMQC-NOESY spectrum of a 1:1 mixture of guest 7 with $\beta-C D$ (middle and right). Detailed comment may be found in the text. Signals of host and guest nuclei are labelled as $\beta-C D$ and $G$, respectively. 


\title{
Unable to Convert Image
}

The dimensions of this image (in pixels) are too large to be converted. For this image to convert, the total number of pixels (height $x$ width) must be less than 40,000,000 (40 megapixels).

\begin{abstract}
A portion of the NOESY spectrum of a $1: 1$ mixture of guest 14 with $\beta-C D$ (left); A portion of the gsHMQC-NOESY spectrum of a 1:1 mixture of guest 7 with $\beta-C D$ (middle and right). Detailed comment may be found in the text. Signals of host and guest nuclei are labelled as $\beta-C D$ and $G$, respectively.
\end{abstract}




\section{Unable to Convert Image}

The dimensions of this image (in pixels) are too large to be converted. For this image to convert, the total number of pixels (height $x$ width) must be less than 40,000,000 (40 megapixels).

Minimised structures of complexes of $\beta-C D$ with amine 26 and 14, respectively. 


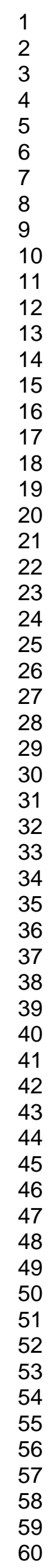

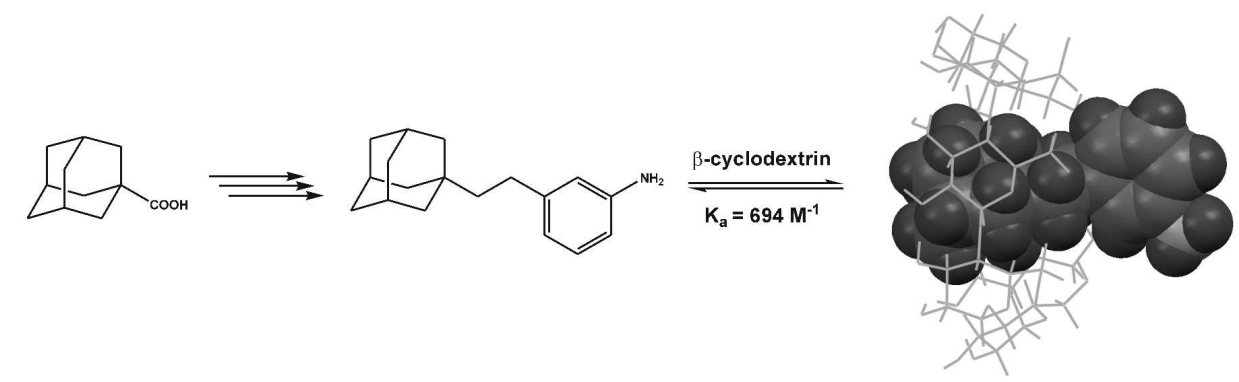

29

32

33

34

35

36

39

40

41

42

43

44

45
46

47

48

49

50 
Overlay picture of successively recorded ${ }^{1} \mathrm{H}$ NMR spectra during NMR titration is shown in Figure S3. Whereas the complexation induced shifts (CIS) of host protons signals are imperceptible, the down-field CIS for guest H4-6 and up-field CIS for H1, H2, H14, and H1618 may be observed.

NMR-based estimation of association constant is shown in following figures. The Job plot for guest 14 (protons H5) is shown in Figure S1. The position of maximum indicates the 1:1 stoichiometry of complex. Titration curves obtained for protons H5 and H1 together with standard least-square regressions are shown in Figure S2. Measurements were carried out in DMSO $-d_{6} / \mathrm{D}_{2} \mathrm{O} 3 / 1 \mathrm{v} / \mathrm{v}$. Solution of guest $(22 \mathrm{mM})$ was titrated with $74 \mathrm{mM}$ stock solution of host at $303 \mathrm{~K}$.

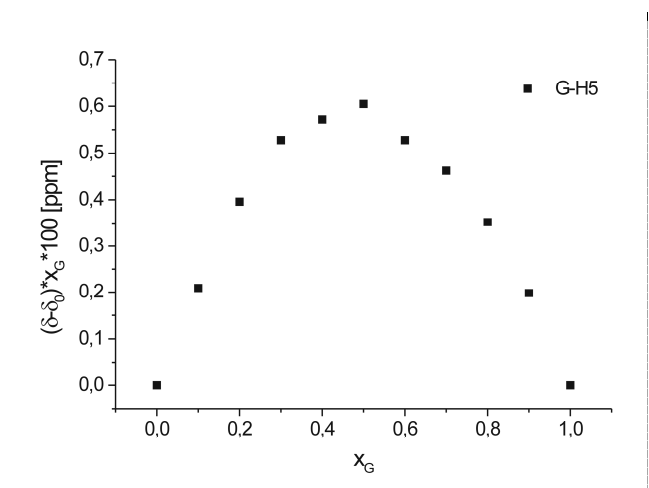

Figure S1

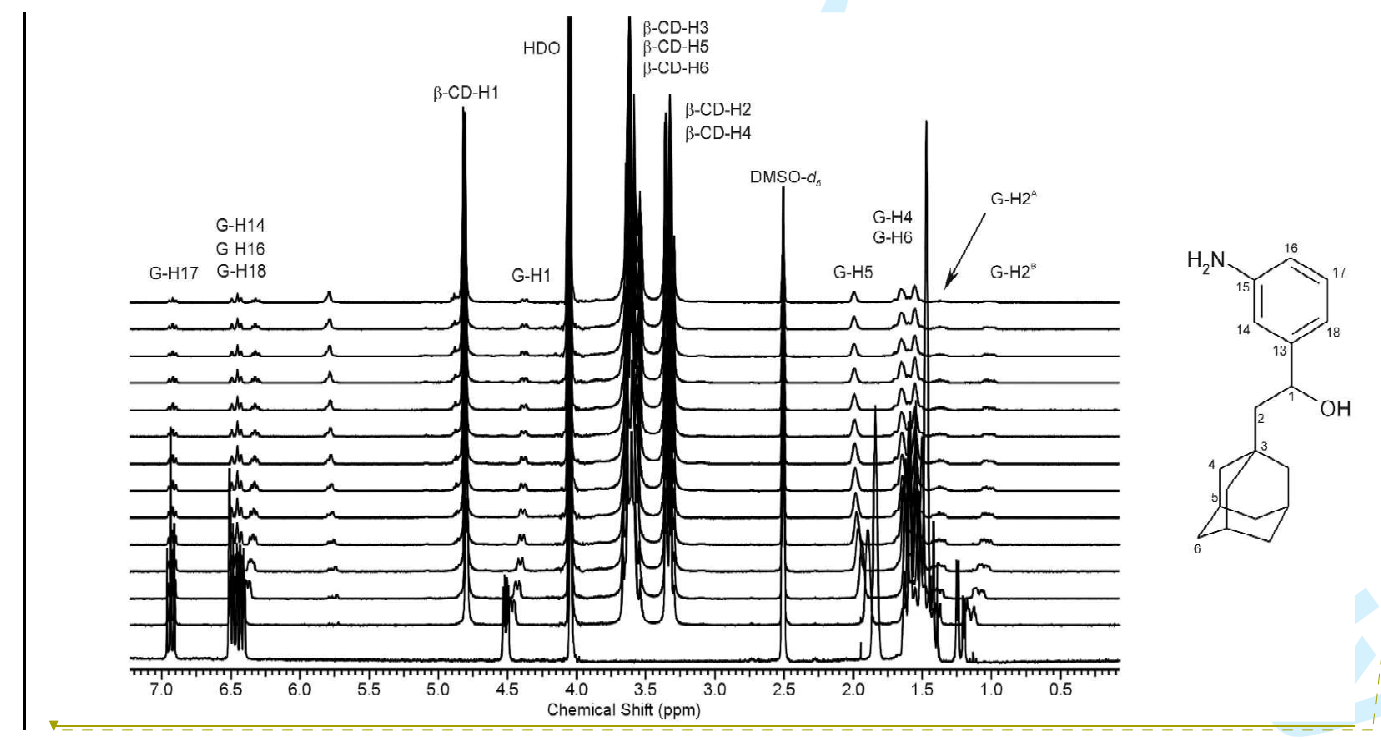

Figure S3

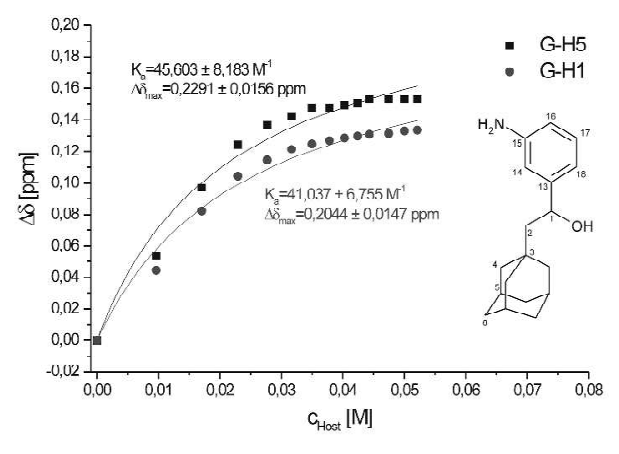

Figure S2

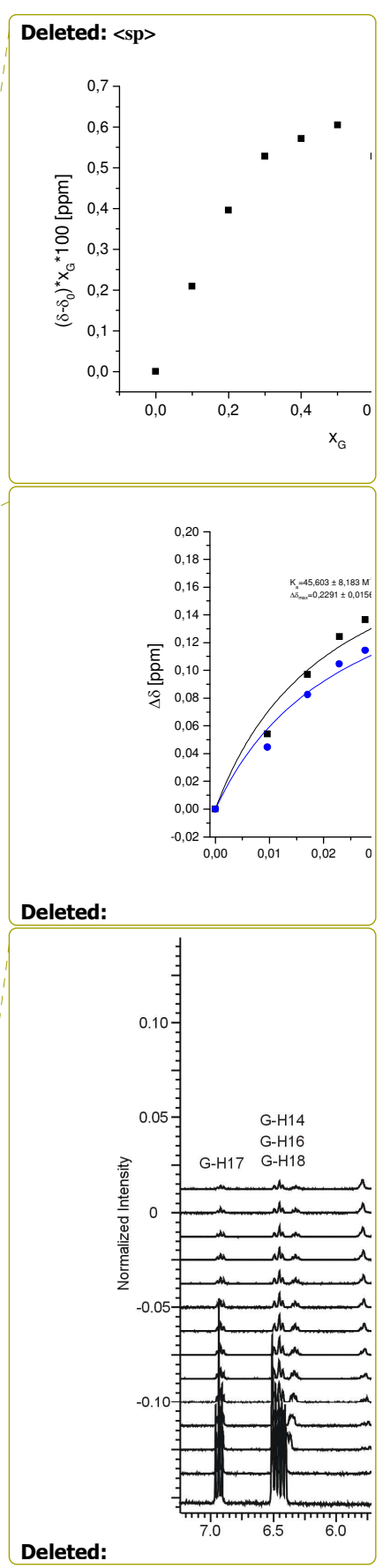


Isothermal titration calorimetry measurements were performed using MicroCal VP-ITC instrument. In individual titrations, injections of $10 \mu \mathrm{L}$ of guest were added from $250 \mu \mathrm{L}$ syringe at an interval of 210 seconds into solution of $\beta$-cyclodextrin in water/DMSO $(1 / 3, \mathrm{v} / \mathrm{v})$ mixture. Typical result of isothermal titration calorimetry experiment for guest $\mathbf{7}$ is shown in Figure S4

Time (min)

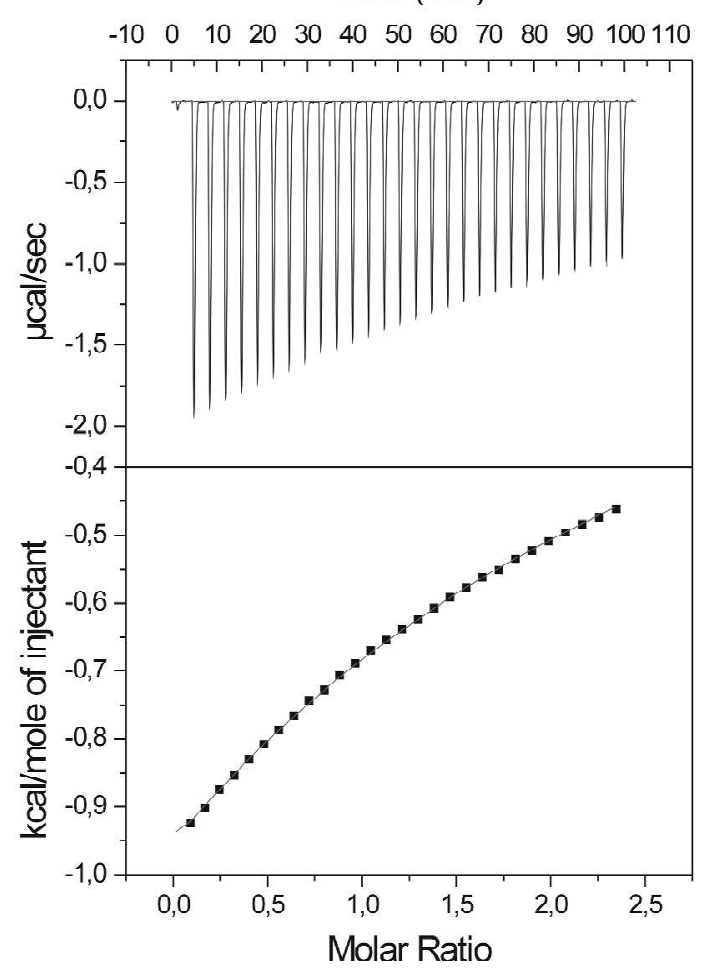

Figure S4

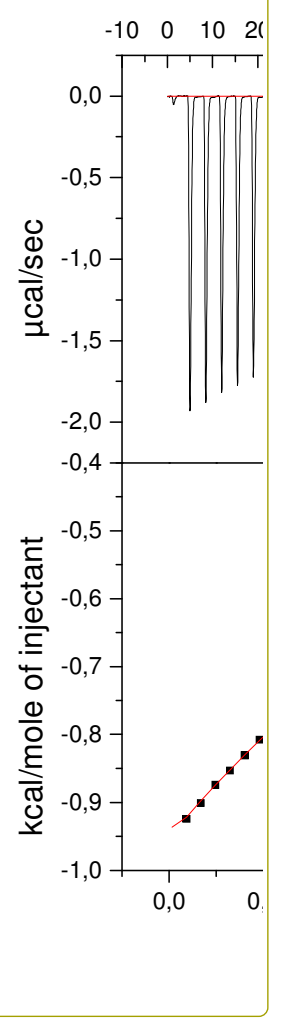

